\title{
SPEH REPRESENTATIONS ARE RELATIVELY DISCRETE
}

\author{
JERROD MANFORD SMITH
}

\begin{abstract}
Let $F$ be a $p$-adic field of characteristic zero and odd residual characteristic. Let $\mathbf{S p}_{2 n}(F)$ denote the symplectic group defined over $F$, where $n \geq 2$. We prove that the Speh representations $U(\delta, 2)$, where $\delta$ is a discrete series representation of $\mathbf{G L}_{n}(F)$, lie in the discrete spectrum of the $p$-adic symmetric space $\mathbf{S p}_{2 n}(F) \backslash \mathbf{G} \mathbf{L}_{2 n}(F)$.
\end{abstract}

\section{Contents}

1. Introduction

2. Notation and terminology

3. Speh representations 531

4. $X$-distinguished Arthur parameters 532

5. Tori and parabolic subgroups: Structure of $\mathbf{S p}_{2 n}(F) \backslash \mathbf{G} \mathbf{L}_{2 n}(F)$

6. Application of the relative Casselman criterion $\quad 542$

Acknowledgments

References $\quad 549$

\section{INTRODUCTION}

Let $F$ be a nonarchimedean local field of characteristic zero and odd residual characteristic $p$. Let $G=\mathbf{G L}_{2 n}(F)$ be an even rank general linear group and let $H=\mathbf{S p}_{2 n}(F)$ be the symplectic group. This paper is concerned with the harmonic analysis on the $p$-adic symmetric space $X=H \backslash G$. We prove that the Speh representations $\mathcal{U}(\delta, 2)$ appear in the discrete spectrum of $X$, as predicted by the conjectures of Sakellaridis and Venkatesh SV17. Our main result, Theorem 6.1 is an unpublished result of $\mathrm{H}$. Jacquet. We frame this result within the construction of (relative) discrete series representations for symmetric quotients of general linear groups carried out in Smi18b Smi18a. The present work relies on the substantial contributions of Heumos and Rallis [HR90, and Offen and Sayag OS07, OS08a, OS08b] in the study of symplectic periods for the general linear group.

All representations are assumed to be on complex vector spaces. In general, a smooth representation $(\pi, V)$ of $G$ is relevant to the harmonic analysis on $X=H \backslash G$ if and only if there exists a nonzero $H$-invariant linear form on the space $V$. If there exists a nonzero element $\lambda$ of $\operatorname{Hom}_{H}(\pi, 1)$, then $(\pi, V)$ is $H$-distinguished. Let $(\pi, V)$ be an irreducible admissible representation of $\mathbf{G L}_{2 n}(F)$. Heumos and Rallis proved that the dimension of the space of $\mathbf{S p}_{2 n}(F)$-invariant linear forms on

Received by the editors August 3, 2018, and, in revised form, July 9, 2020.

2010 Mathematics Subject Classification. Primary 22E50; Secondary 22E35.

Key words and phrases. Relative discrete series, distinguished representation, symplectic group, Speh representation. 
$V$ is at most one [HR90, Theorem 2.4.2]. In addition, Heumos and Rallis showed that any irreducible admissible representation of $\mathbf{G L}_{2 n}(F)$ cannot be both generic and $\mathbf{S p}_{2 n}(F)$-distinguished. Recall that representation of $\mathbf{G} \mathbf{L}_{n}(F)$ is generic if it admits a Whittaker model (see Rod73 for more information on Whittaker models).

To see that an $H$-distinguished smooth representation $(\pi, V)$ of $G$ occurs in the space $C^{\infty}(X)$ of smooth (locally constant) functions on $X=H \backslash G$ one considers its relative matrix coefficients. Let $\lambda \in \operatorname{Hom}_{H}(\pi, 1)$ be nonzero. For any $v \in V$, define a function $\varphi_{\lambda, v}$ by declaring that $\varphi_{\lambda, v}(H g)=\langle\lambda, \pi(g) v\rangle$. The functions $\varphi_{\lambda, v}$ are smooth, since $\pi$ is smooth, and well-defined because $\lambda$ is $H$-invariant. Moreover, the map that sends $v \in V$ to the $\lambda$-relative matrix coefficient $\varphi_{\lambda, v}$ intertwines $(\pi, V)$ and the right regular representation of $G$ on $C^{\infty}(X)$. It is a fundamental problem to determine which irreducible representations of $G$ occur in the space $L^{2}(X)$ of square integrable functions on $X$. The discrete spectrum $L_{\text {disc }}^{2}(X)$ of $X$ is the direct sum of all irreducible $G$-subrepresentations of the space $L^{2}(X)$ of square integrable functions on $X$. We prove, in Theorem 6.1, that the Speh representations $\mathcal{U}(\delta, 2)$ appear in $L_{\text {disc }}^{2}\left(\mathbf{S p}_{2 n}(F) \backslash \mathbf{G L}_{2 n}(F)\right)$. On the other hand, we do not prove that such representations are the only discrete series; we face the same obstacles discussed in Smi18b, Remark 6.6].

Sakellaridis and Venkatesh have developed a framework ecnompassing the study of harmonic analysis on $p$-adic symmetric spaces and its deep connections with periods of automorphic forms and Langlands functoriality [SV17. In addition to providing explicit Plancherel formulas, Sakellaridis and Venkatesh have made precise conjectures describing the Arthur parameters of representations in the discrete series of symmetric spaces (and, more generally, spherical varieties) SV17, Conjectures 1.3.1 and 16.2.2]. In fact, their conjectures predict that the discrete series of $\mathbf{S p}_{2 n}(F) \backslash \mathbf{G L}_{2 n}(F)$ consists precisely of the Speh representations.

We conclude the introduction with a summary of the contents of the paper. In Section 2 we establish notation regarding $p$-adic symmetric spaces and representations; in addition, we review the Relative Casselman Criterion established by Kato and Takano [KT10]. We review the construction of the Speh representation in Section 3. In Section 4, we review the conjectures of Sakellaridis and Venkatesh and we demonstrate that their work predicts that the Speh representations $\mathcal{U}(\delta, 2)$ should appear in the discrete spectrum of $\mathbf{S p}_{2 n}(F) \backslash \mathbf{G} \mathbf{L}_{2 n}(F)$ (see Proposition 4.5). We determine the fine structure of the symmetric space $\mathbf{S p}_{2 n}(F) \backslash \mathbf{G L}_{2 n}(F)$ in Section 5 in particular, we realize the group $\mathbf{S p}_{2 n}(F)$ as the fixed points of an involution $\theta$ on $\mathbf{G L}_{2 n}(F)$, and determine the restricted root system and maximal $\theta$-split parabolic subgroups of $\mathbf{G L}_{2 n}(F)$ relative to $\theta$. In Section 6 we prove our main result, Theorem 6.1, by applying the Relative Casselman Criterion (see Theorem 2.7).

In Section 5.2, we make an effort to set the present work within the program started in Smi18b Smi18a, where relative discrete series representations have been systematically constructed via parabolic induction from distinguished discrete series representations of $\theta$-elliptic Levi subgroups. In fact, we realize the Speh representations as quotients of representations induced from distinguished discrete series of certain maximal $\theta$-elliptic Levi subgroups. The present setting is complicated by the fact that representations induced from discrete series are generic and therefore not distinguished by the symplectic group. In particular, although we expect that the construction of relative discrete series carried out in Smi18b, Smi18a should 
generalize, some care must be taken to handle the "disjointness-of-models" phenomena as in the case of the Whittaker and symplectic models HR90, Theorem 3.2.2], and Klyachko models OS08b].

\section{Notation AND TERMinOLOGY}

Let $F$ be a nonarchimedean local field of characteristic zero and odd residual characteristic $p$. Let $\mathcal{O}_{F}$ be the ring of integers of $F$. Fix a uniformizer $\varpi$ of $F$. Let $q$ be the cardinality of the residue field $k_{F}$ of $F$. Let $|\cdot|_{F}$ denote the normalized absolute value on $F$ such that $|\varpi|_{F}=q^{-1}$. We reserve the notation $|\cdot|$ for the usual absolute value on $\mathbb{C}$.

2.1. Reductive groups and $p$-adic symmetric spaces. Let $\mathbf{G}$ be a connected reductive group defined over $F$. Let $\theta$ be an $F$-involution of $\mathbf{G}$. Let $\mathbf{H}=\mathbf{G}^{\theta}$ be the subgroup of $\theta$-fixed points in $\mathbf{G}$. Write $G=\mathbf{G}(F)$ for the group of $F$-points of G, similarly $H=\mathbf{H}(F)$. The quotient $H \backslash G$ is a $p$-adic symmetric space. We will routinely abuse notation and identify an algebraic $F$-variety $\mathbf{X}$ with its $F$-points $X=\mathbf{X}(F)$. When the distinction is to be made, we will use boldface to denote the algebraic variety and regular typeface for the set of $F$-points.

For an $F$-torus $\mathbf{A} \subset \mathbf{G}$, let $A^{1}$ be the subgroup $\mathbf{A}\left(\mathcal{O}_{F}\right)$ of $\mathcal{O}_{F}$-points of $A=$ $\mathbf{A}(F)$. We use $Z_{G}$ to denote the centre of $G$ and $A_{G}$ to denote the $F$-split component of the centre of $G$. Let $X^{*}(G)$ denote the group of $F$-rational characters of the algebraic group G. If $Y$ is a subset of a group $G$, then let $N_{G}(Y)$ denote the normalizer of $Y$ in $G$ and let $C_{G}(Y)$ denote the centralizer of $Y$ in $G$.

2.1.1. Tori and root systems relative to involutions. An element $g \in G$ is $\theta$-split if $\theta(g)=g^{-1}$. An $F$-torus $S$ contained in $G$ is $(\theta, F)$-split if $S$ is $F$-split and every element of $S$ is $\theta$-split.

Let $S_{0}$ be a maximal $(\theta, F)$-split torus of $G$. Let $A_{0}$ be a $\theta$-stable maximal $F$ split torus of $G$ that contains $S_{0}$ [HW93, Lemma 4.5(iii)]. Let $\Phi_{0}=\Phi\left(G, A_{0}\right)$ be the root system of $G$ with respect to $A_{0}$. Let $W_{0}=W\left(G, A_{0}\right)=N_{G}\left(A_{0}\right) / C_{G}\left(A_{0}\right)$ be the Weyl group of $G$ with respect to $A_{0}$.

The torus $A_{0}$ is $\theta$-stable, so there is an action of $\theta$ on the $F$-rational characters $X^{*}\left(A_{0}\right)$; moreover, $\Phi_{0}$ is a $\theta$-stable subset of $X^{*}\left(A_{0}\right)$. Recall that a base of $\Phi_{0}$ determines a choice of positive roots $\Phi_{0}^{+}$.

Definition 2.1. A base $\Delta_{0}$ of $\Phi_{0}$ is called a $\theta$-base if for every positive root $\alpha \in \Phi_{0}^{+}$ such that $\theta(\alpha) \neq \alpha$ we have that $\theta(\alpha) \in \Phi_{0}^{-}=-\Phi_{0}^{+}$.

Let $\Delta_{0}$ be a $\theta$-base of $\Phi_{0}$. Let $r: X^{*}\left(A_{0}\right) \rightarrow X^{*}\left(S_{0}\right)$ be the surjective map defined by restriction of $\left(F\right.$-rational) characters. Define $\bar{\Phi}_{0}=r\left(\Phi_{0}\right) \backslash\{0\}$ and $\bar{\Delta}_{0}=r\left(\Delta_{0}\right) \backslash\{0\}$. The set $\bar{\Phi}_{0}$ coincides with $\Phi_{0}\left(G, S_{0}\right)$ and is referred to as the restricted root system of $H \backslash G$ [HW93, Proposition 5.9]. The set $\bar{\Delta}_{0}$ is a base of the root system $\bar{\Phi}_{0}$. Note that $\bar{\Phi}_{0}$ is not necessarily reduced. Let $\Phi_{0}^{\theta}$ and $\Delta_{0}^{\theta}$ be the subsets of $\theta$-fixed roots in $\Phi_{0}$, respectively $\Delta_{0}$. Observe that $\bar{\Phi}_{0}=r\left(\Phi_{0} \backslash \Phi_{0}^{\theta}\right)$ and $\bar{\Delta}_{0}=r\left(\Delta_{0} \backslash \Delta_{0}^{\theta}\right)$.

Let $\bar{\Theta}$ be a subset of $\bar{\Delta}_{0}$. Set $[\bar{\Theta}]=r^{-1}(\bar{\Theta}) \cup \Delta_{0}^{\theta}$. Subsets of $\Delta_{0}$ of the form $[\bar{\Theta}]$ are called $\theta$-split. Maximal $\theta$-split subsets of $\Delta_{0}$ are of the form $\left[\bar{\Delta}_{0} \backslash\{\bar{\alpha}\}\right]$, where $\bar{\alpha} \in \bar{\Delta}_{0}$. 
2.1.2. Parabolic subgroups relative to involutions. Let $\mathbf{P}$ be an $F$-parabolic subgroup of $\mathbf{G}$. We refer to an $F$-parabolic subgroup of $\mathbf{G}$ simply as a parabolic subgroup. Let $\mathbf{N}$ be the unipotent radical of $\mathbf{P}$. The reductive quotient $\mathbf{M} \cong \mathbf{P} / \mathbf{N}$ is called a Levi factor of $\mathbf{P}$. We denote by $\delta_{P}$ the modular character of $P=\mathbf{P}(F)$ given by $\delta_{P}(p)=\left|\operatorname{det} \operatorname{Ad}_{\mathfrak{n}}(p)\right|_{F}$, where $\mathfrak{n}$ is the Lie algebra of $\mathbf{N}$.

Let $M$ be a Levi subgroup of $G$. Let $A_{M}$ denote the $F$-split component of the centre of $M$. The $(\theta, F)$-split component of $M$, denoted by $S_{M}$, is the largest $(\theta, F)$-split torus of $M$ that is contained in $A_{M}$. More precisely,

$$
S_{M}=\left(\left\{a \in A_{M}: \theta(a)=a^{-1}\right\}\right)^{\circ},
$$

where $(\cdot)^{\circ}$ denotes the Zariski-connected component of the identity.

Definition 2.2. A parabolic subgroup $P$ of $G$ is $\theta$-split if $\theta(P)$ is opposite to $P$, in which case $M=P \cap \theta(P)$ is a $\theta$-stable Levi subgroup of $P$.

If $\Theta \subset \Delta_{0}$ is $\theta$-split, then the $\Delta_{0}$-standard parabolic subgroup $P_{\Theta}$ is $\theta$-split. Let $\Phi_{\Theta}$ be the subsystem of $\Phi_{0}$ generated by $\Theta$. The standard parabolic subgroup $P_{\Theta}$ has unipotent radical $N_{\Theta}$ generated by the root subgroups $N_{\alpha}$, where $\alpha \in \Phi_{0}^{+} \backslash \Phi_{\Theta}^{+}$. The standard Levi subgroup of $P_{\Theta}$ is $M_{\Theta}$, which is the centralizer in $G$ of the $F$ split torus $A_{\Theta}=\left(\bigcap_{\alpha \in \Theta} \operatorname{ker} \alpha\right)^{\circ}$. Any $\Delta_{0}$-standard $\theta$-split parabolic subgroup of $G$ arises from a $\theta$-split subset of $\Delta_{0}$ [KT08, Lemma 2.5(1)].

Let $\Theta \subset \Delta_{0}$ be $\theta$-split. The $(\theta, F)$-split component of $M_{\Theta}$ is equal to

$$
S_{\Theta}=\left(\bigcap_{\bar{\alpha} \in r(\Theta)} \operatorname{ker}\left(\bar{\alpha}: S_{0} \rightarrow F^{\times}\right)\right)^{\circ} .
$$

For any $0<\epsilon \leq 1$, define

$$
S_{\Theta}^{-}(\epsilon)=\left\{s \in S_{\Theta}:|\alpha(s)|_{F} \leq \epsilon, \text { for all } \alpha \in \Delta_{0} \backslash \Theta\right\} .
$$

We write $S_{\Theta}^{-}$for $S_{\Theta}^{-}(1)$ and refer to $S_{\Theta}^{-}$as the dominant part of $S_{\Theta}$.

By HH98, Theorem 2.9], the $\theta$-split subset $\Delta_{0}^{\theta}$ determines the standard minimal $\theta$-split parabolic subgroup $P_{0}=P_{\Delta_{0}^{\theta}}$. Let $N_{0}$ be the unipotent radical of $P_{0}$. The standard Levi subgroup $M_{0}$ of $P_{0}$ is the centralizer in $G$ of the maximal $(\theta, F)$-split torus $S_{0}$.

Lemma 2.3 ([KT08, Lemma 2.5]). Let $S_{0} \subset A_{0}, \Delta_{0}$, and $P_{0}=M_{0} N_{0}$ be as above.

(1) Any $\theta$-split parabolic subgroup $P$ of $G$ is conjugate to a $\Delta_{0}$-standard $\theta$-split parabolic subgroup by an element $g \in\left(\mathbf{H M}_{0}\right)(F)$.

(2) If the group of $F$-points of the product $\left(\mathbf{H M}_{0}\right)(F)$ is equal to $H M_{0}$, then any $\theta$-split parabolic subgroup of $G$ is $H$-conjugate to a $\Delta_{0}$-standard $\theta$-split parabolic subgroup.

Let $P=M N$ be a $\theta$-split parabolic subgroup. Pick $g \in\left(\mathbf{H M}_{0}\right)(F)$ such that $P=g P_{\Theta} g^{-1}$ for some $\theta$-split subset $\Theta \subset \Delta_{0}$. Since $g \in\left(\mathbf{H M}_{0}\right)(F)$ we have that $g^{-1} \theta(g) \in \mathbf{M}_{0}(F)$, and we have $S_{M}=g S_{\Theta} g^{-1}$. For a given $\epsilon>0$, one may extend the definition of $S_{\Theta}^{-}$in (2.1) to the torus $S_{M}$. Set $S_{M}^{-}(\epsilon)=g S_{\Theta}^{-}(\epsilon) g^{-1}$ and define $S_{M}^{-}=S_{M}^{-}(1)$. Write $S_{M}^{1}$ to denote the group of $\mathcal{\Theta}_{F}$-points $S_{M}\left(\mathcal{\Theta}_{F}\right)$. 
2.2. Distinguished representations and relative matrix coefficients. A representation $(\pi, V)$ of $G$ is smooth if for every $v \in V$ the stabilizer of $v$ in $G$ is an open subgroup. A smooth representation $(\pi, V)$ of $G$ is admissible if, for every compact open subgroup $K$ of $G$, the subspace $V^{K}$ of $K$-invariant vectors is finite dimensional. All of the representations that we consider are smooth and admissible. A quasi-character of $G$ is a one-dimensional representation. Let $(\pi, V)$ be a smooth representation of $G$. If $\omega$ is a quasi-character of $Z_{G}$, then $(\pi, V)$ is called an $\omega$-representation if $\pi$ has central character $\omega$.

Let $P$ be a parabolic subgroup of $G$ with Levi subgroup $M$ and unipotent radical $N$. Given a smooth representation $\left(\rho, V_{\rho}\right)$ of $M$ we may inflate $\rho$ to a representation of $P$, also denoted $\rho$, by declaring that $N$ acts trivially. We define the representation $\iota_{P}^{G} \rho$ of $G$ to be the (normalized) parabolically induced representation $\operatorname{Ind}_{P}^{G}\left(\delta_{P}^{1 / 2} \otimes \rho\right)$. We will also use the Bernstein-Zelevinsky [BZ77, Zel80] notation $\pi_{1} \times \ldots \times \pi_{k}$ for the (normalized) parabolically induced representation $\left.\iota_{P_{\left(m_{1}, \ldots, m_{k}\right)}} \mathbf{G L}_{m}(F) \ldots \ldots \otimes \pi_{k}\right)$ of $\mathbf{G L}_{m}(F)$ obtained from the standard (block-upper triangular) parabolic subgroup $P_{\left(m_{1}, \ldots, m_{k}\right)}$ and representations $\pi_{j}$ of $\mathbf{G L}_{m_{j}}(F)$, where $\sum_{j=1}^{k} m_{j}=m$.

Let $(\pi, V)$ be a smooth representation of $G$. Let $\left(\pi_{N}, V_{N}\right)$ denote the normalized Jacquet module of $\pi$ along $P$. Precisely, $V_{N}$ is the quotient of $V$ by the $P$-stable subspace $V(N)=\operatorname{span}\{\pi(n) v-v: n \in N, v \in V\}$, and the action of $P$ on $V_{N}$ is normalized by $\delta_{P}^{-1 / 2}$. The unipotent radical $N$ of $P$ acts trivially on $\left(\pi_{N}, V_{N}\right)$, and we will regard $\left(\pi_{N}, V_{N}\right)$ as a representation of the Levi factor $M \cong P / N$ of $P$.

We also let $\pi$ denote its restriction to $H$. Let $\chi$ be a quasi-character of $H$.

Definition 2.4. The representation $\pi$ is $(H, \chi)$-distinguished if the space $\operatorname{Hom}_{H}(\pi, \chi)$ is nonzero. If $\pi$ is $(H, 1)$-distinguished, where 1 is the trivial character of $H$, then we will simply call $\pi H$-distinguished.

Let $(\pi, V)$ be a smooth $H$-distinguished $\omega$-representation of $G$. Note that $\omega$ must be trivial on $Z_{G} \cap H$. Let $\lambda \in \operatorname{Hom}_{H}(\pi, 1)$ be a nonzero $H$-invariant linear functional on $V$. Let $v \in V$ be a nonzero vector. Define the $\lambda$-relative matrix coefficient associated to $v$ to be the complex valued function $\varphi_{\lambda, v}: G \rightarrow \mathbb{C}$ given by $\varphi_{\lambda, v}(g)=\langle\lambda, \pi(g) v\rangle$. When $\lambda$ is understood, we drop it from the terminology and refer to the relative matrix coefficients of $\pi$. Since $(\pi, V)$ is assumed to be smooth, for all $v \in V$, the function $\varphi_{\lambda, v}$ lies in the space $C^{\infty}(G)$ of smooth (that is, locally constant) $\mathbb{C}$-valued functions on $G$. Moreover, since $\pi$ is an $\omega$-representation, the functions $\varphi_{\lambda, v}$ lie in the subspace $C_{\omega}^{\infty}(G)$ consisting of smooth functions $f: G \rightarrow \mathbb{C}$ such that $f(z g)=\omega(z) f(g)$, for all $z \in Z_{G}$ and $g \in G$. Observe that, since $\lambda$ is $H$-invariant, for all $g \in G, z \in Z_{G}$, and $h \in H$ we have

$$
\begin{aligned}
\varphi_{\lambda, v}(h z g) & =\langle\lambda, \pi(h z g) v\rangle \\
& =\omega(z)\langle\lambda, \pi(g) v\rangle \\
& =\omega(z) \varphi_{\lambda, v}(g) .
\end{aligned}
$$

For any $v \in V$, the $\lambda$-relative matrix coefficient $\varphi_{\lambda, v}$ descends to well a defined function on $H \backslash G$ and satisfies $\varphi_{\lambda, v}(H z g)=\omega(z) \varphi_{\lambda, v}(H g)$, for $z \in Z_{G}$ and $H g \in$ $H \backslash G$. Further assume that the central character $\omega$ of $(\pi, V)$ is unitary. In this case, the function $Z_{G} H \cdot g \mapsto\left|\varphi_{\lambda, v}(g)\right|$ is well defined on $Z_{G} H \backslash G$. The centre $Z_{G}$ of $G$ is unimodular since it is abelian. The fixed point subgroup $H$ is also reductive and thus unimodular. By [Rob83, Proposition 12.8], there exists a $G$-invariant measure on the quotient $Z_{G} H \backslash G$. 
Definition 2.5. Let $\omega$ be a unitary character of $Z_{G}$. Let $(\pi, V)$ be an $H$-distinguished $\omega$-representation of $G$. Then $(\pi, V)$ is said to be

(1) $(H, \lambda)$-relatively square integrable if and only if all of the $\lambda$-relative matrix coefficients are square integrable modulo $Z_{G} H$.

(2) $H$-relatively square integrable if and only if $\pi$ is $(H, \lambda)$-relatively square integrable for every $\lambda \in \operatorname{Hom}_{H}(\pi, 1)$.

2.3. Exponents and the relative Casselman criterion. Let $(\pi, V)$ be a finitely generated admissible representation of $G$. Let $\varepsilon_{x} p_{Z_{G}}(\pi)$ be the (finite) set of quasicharacters of $Z_{G}$ that occur as the central characters of the irreducible subquotients of $\pi$. We refer to the characters that appear in $\varepsilon x p_{Z_{G}}(\pi)$ as the exponents of $\pi$. By Cas95, Proposition 2.1.9], the quasi-character $\chi: Z_{G} \rightarrow \mathbb{C}^{\times}$occurs in $\mathscr{E} x p_{Z_{G}}(\pi)$ if and only if the generalized $\chi$-eigenspace for the action of $Z_{G}$ on $V$ is nonzero. Let $Z$ be a closed subgroup of $Z_{G}$. The exponents $E x p_{Z}(\pi)$ with respect to the action of $Z$ on $V$ are defined analogously. If $Z_{1} \supset Z_{2}$ are two closed subsets of $Z_{G}$, then the map $\mathscr{E} x p_{Z_{1}}(\pi) \rightarrow \mathscr{E} x p_{Z_{2}}(\pi)$ defined by restriction of quasi-characters is surjective (see, for instance, [Smi18b, Lemma 4.15]).

Let $P=M N$ be a parabolic subgroup of $G$ with unipotent radical $N$ and Levi factor $M$. The normalized Jacquet module $\left(\pi_{N}, V_{N}\right)$ of $(\pi, V)$ along $P$ is also finitely generated and admissible Cas95, Theorem 3.3.1]. The set $\mathscr{E} x p_{A_{M}}\left(\pi_{N}\right)$ of exponents of $\pi_{N}$, with respect to the action of the $F$-split component $A_{M}$ of $M$, is referred to as the set of exponents of $\pi$ along $P$.

Lemma 2.6 ([Smi18b, Lemma 4.16]). Let $P=M N$ be a parabolic subgroup of $G$, let $(\rho, W)$ be a finitely generated admissible representation of $M$, and let $\pi=\iota_{P}^{G} \rho$. The exponents $E_{x p_{A_{G}}}(\pi)$ of $\pi$ are the restrictions to $A_{G}$ of the exponents $\mathscr{E x p}_{A_{M}}(\rho)$ of $\rho$.

Let $(\pi, V)$ be a finitely generated admissible $H$-distinguished representation of $G$. Let $\lambda \in \operatorname{Hom}_{H}(\pi, 1)$ be a nonzero $H$-invariant linear form on $(\pi, V)$. In [KT10], Kato and Takano defined

$$
\operatorname{Exp}_{Z}(\pi, \lambda)=\left\{\chi \in \mathscr{E x p}_{Z}(\pi):\left.\lambda\right|_{V_{\chi}} \neq 0\right\},
$$

for any closed subgroup $Z$ of $Z_{G}$, where

$$
V_{\chi}=\bigcup_{n=1}^{\infty}\left\{v \in V:(\pi(z)-\chi(z))^{n} v=0, \forall z \in Z\right\}
$$

is the generalized $\chi$-eigenspace for the $Z$ action on $V$. The elements of $\varepsilon x p_{A_{G}}(\pi, \lambda)$ are referred to as the exponents of $\pi$ relative to $\lambda$. Let $P$ be a $\theta$-split parabolic subgroup of $G$ with unipotent radical $N$ and $\theta$-stable Levi subgroup $M=P \cap \theta(P)$. Using Casselman's Canonical Lifting [Cas95, Proposition 4.1.4], Kato-Takano KT08 and Lagier Lag08 defined an $M^{\theta}$-invariant linear functional $\lambda_{N} \in \operatorname{Hom}_{M^{\theta}}\left(\pi_{N}, 1\right)$, canonically associated to $\lambda$, on the Jacquet module $\pi_{N}$ of $\pi$ along $P$. We refer the reader to [KT08, Proposition 5.6] for details of the construction and additional properties of the map $\lambda \mapsto \lambda_{N}$. We may now state the Relative Casselman Criterion [KT10, Theorem 4.7].

Theorem 2.7 (Relative Casselman Criterion). Let $\omega$ be a unitary character of $Z_{G}$. Let $(\pi, V)$ be a finitely generated admissible $H$-distinguished $\omega$-representation 
of $G$. Fix a nonzero element $\lambda$ in $\operatorname{Hom}_{H}(\pi, V)$. The representation $(\pi, V)$ is $(H, \lambda)$ relatively square integrable if and only if the condition

$$
|\chi(s)|<1, \quad \forall \chi \in \mathcal{E} x p_{S_{M}}\left(\pi_{N}, \lambda_{N}\right), \forall s \in S_{M}^{-} \backslash S_{G} S_{M}^{1}
$$

is satisfied for every proper $\theta$-split parabolic subgroup $P=M N$ of $G$.

2.4. Conventions regarding $\mathbf{S p}_{2 n}(F) \backslash \mathbf{G L}_{2 n}(F)$. From now on, unless otherwise specified, we let $G=\mathbf{G L}_{2 n}(F)$ and let $H=\mathbf{S p}_{2 n}(F)$, where $n \geq 2$. We will realize the symplectic group $H$ explicitly as the subgroup of $G$ fixed pointwise by the involution $\theta$ given by

$$
\theta(g)=\varepsilon_{2 n}^{-1 t} g^{-1} \varepsilon_{2 n},
$$

where ${ }^{t} g$ denotes the transpose of $g \in G$,

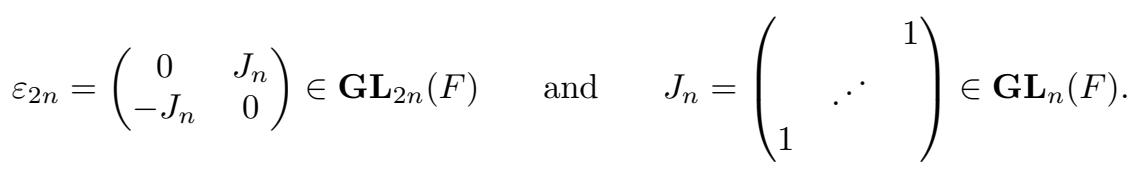

Note that $\varepsilon_{2 n}$ is a nonsingular skew-symmetric element of $G$; moreover, $\varepsilon_{2 n}^{-1}={ }^{t} \varepsilon_{2 n}$. With this choice of involution, the subgroup of upper triangular matrices in $H$ is a Borel subgroup (over $F$ ). Let $A_{0}$ be the maximal diagonal $F$-split torus of $G$.

There is a right $G$-action on the set of involutions of $G$ given by

$$
g \cdot \theta(x)=g^{-1} \theta\left(g x g^{-1}\right) g,
$$

for any $x, g \in G$. Any involution of the form $g \cdot \theta$ is said to be $G$-equivalent to $\theta$. If $x \in G$ is skew-symmetric, then we obtain a realization of the symplectic group as the fixed points of the involution $\theta_{x}$ defined by

$$
\theta_{x}(g)=x^{-1 t} g^{-1} x .
$$

Moreover, the $G$-action on involutions is compatible with the right $G$-action on the set of skew-symmetric matrices given by $x \cdot g={ }^{t} g x g$, for any $g \in G$ and any skew-symmetric matrix $x \in G$. Indeed, if $x, g \in G$ and $x$ is skew-symmetric, then $g \cdot \theta_{x}=\theta_{x \cdot g}$.

We will write $\operatorname{diag}\left(a_{1}, \ldots, a_{m}\right)$ to denote the diagonal $m \times m$ matrix with entries $a_{1}, \ldots, a_{m}$ on the main diagonal. Given a partition $\left(m_{1}, \ldots, m_{k}\right)$ of a positive integer $m$, write $P_{\left(m_{1}, \ldots, m_{k}\right)}$ for the standard block-upper triangular parabolic subgroup of $\mathbf{G L}_{m}(F)$ with Levi factor $M_{\left(m_{1}, \ldots, m_{k}\right)}$ and unipotent radical $N_{\left(m_{1}, \ldots, m_{k}\right)}$. Write $\nu$ for the unramified character $|\operatorname{det}(\cdot)|_{F}$ of $\mathbf{G L}_{m}(F)$, where $m$ is understood from context.

\section{SPEH REPRESENTATIONS}

Recall that a representation $\pi$ of $\mathbf{G L}_{m}(F)$ is said to be generic if it admits a Whittaker model, that is, if there exists a nonzero intertwining operator in the space $\operatorname{Hom}_{N_{m}}\left(\pi, \operatorname{Ind}_{N_{m}}^{\mathbf{G L}_{m}(F)} \Psi_{m}\right)$, where $N_{m}$ is the subgroup of $\mathbf{G L}_{m}(F)$ consisting of upper triangular unipotent matrices and $\Psi_{m}$ is a non-degenerate character of $N_{m}$

Let $\delta$ be an irreducible square integrable representation of $\mathbf{G L}_{n}(F)$. The parabolically induced representation $\nu^{1 / 2} \delta \times \nu^{-1 / 2} \delta$ has length two and admits a unique irreducible generic subrepresentation $\mathcal{Z}(\delta, 2)$ and a unique irreducible quotient $\mathcal{U}(\delta, 2)$ 
[BZ77, Zel80. In particular, we have the following short exact sequence of $G$ representations

$$
0 \rightarrow \mathcal{Z}(\delta, 2) \rightarrow \nu^{1 / 2} \delta \times \nu^{-1 / 2} \delta \rightarrow \mathcal{U}(\delta, 2) \rightarrow 0 .
$$

The representations $\mathcal{U}(\delta, 2)$ are the Speh representations.

Heumos and Rallis proved that the Speh representations $\mathcal{U}(\delta, 2)$ are $H$-distinguished by constructing a nonzero $H$-invariant linear functional on the full induced representation $\nu^{1 / 2} \delta \times \nu^{-1 / 2} \delta$ and then appealing to HR90, Theorem 3.2.2] to show that the generic subrepresentation $\mathcal{Z}(\delta, 2)$ cannot be $H$-distinguished. One can then use the exact sequence (3.1) to conclude that the invariant functional on $\nu^{1 / 2} \delta \times \nu^{-1 / 2} \delta$ descends to a well-defined nonzero $H$-invariant linear functional on $\mathcal{U}(\delta, 2)$. Note that the invariant form on $\nu^{1 / 2} \delta \times \nu^{-1 / 2} \delta$ may be realized via Off17, Proposition 7.1], (cf. [Smi20, Lemma 1.3.4]).

Next, let us recall descriptions of the generalized Steinberg representations and generalized Speh representations of general linear groups. Let $\rho$ be an irreducible unitary supercuspidal representation of $\mathbf{G L}_{r}(F), r \geq 1$. Let $k \geq 2$ be an integer. By [Zel80, Theorem 9.3], the induced representation

$$
\nu^{\frac{k-1}{2}} \rho \times \ldots \times \nu^{\frac{1-k}{2}} \rho
$$

of $\mathbf{G L}_{k r}(F)$ admits a unique irreducible subrepresentation $\mathcal{Z}(\rho, k)$; moreover, $\mathcal{Z}(\rho, k)$ is square integrable. Often in the literature, $\mathcal{Z}(\rho, k)$ is denoted by $\operatorname{St}(\rho, k)$ and these are the generalized Steinberg representations of $\mathbf{G L}_{k r}(F)$. Let $\delta$ be a discrete series representation of $\mathbf{G L}_{d}(F), d \geq 2$. Let $m \geq 2$ be an integer. By [Zel80, Theorem 6.1(a)], the induced representation

$$
\nu^{\frac{m-1}{2}} \delta \times \ldots \times \nu^{\frac{1-m}{2}} \delta
$$

admits a unique irreducible (unitary) quotient $\mathcal{U}(\delta, m)$.

Remark 3.1. The Speh representations, and generalized Speh representations, feature prominently in the classification of the unitary dual of general linear groups carried out by Tadić Tad85, Tad86.

Remark 3.2. The method used by Heumos and Rallis to demonstrate the $\mathbf{S p}_{2 n}(F)$ distinction of the Speh representations $\mathcal{U}(\delta, 2)$ does not immediately extend to the generalized Speh representations $\mathcal{U}(\delta, m), m>2$. However, Offen and Sayag OS07] study the distinction of the generalized Speh representations by utilizing work of Jacquet and Rallis [JR96] and Bernstein's meromorphic continuation. The method of Offen and Sayag, used to prove the "hereditary property of symplectic periods," is a special case of the method of Blanc and Delorme [BD08]. We refer the reader to OS07] for more details.

\section{4. $X$-Distinguished Arthur parameters}

In the following discussion, $\mathbf{G}$ can be taken to be an arbitrary connected reductive group that is split over $F$. We return to $G=\mathbf{G L}_{2 n}(F)$ in Section 4.1.

Let $\mathcal{W}_{F}$ be the Weil group of $F$ and let $\mathcal{L}_{F}=\mathcal{W}_{F} \times \mathrm{SL}(2, \mathbb{C})$ be the Weil-Deligne group of $F$. Since $\mathbf{G}$ is split over $F, \mathcal{W}_{F}$ acts trivially on the complex dual group $G^{\vee}$, and the $L$-group of $\mathbf{G}$ can be identified with the dual group ${ }^{L} G=G^{\vee}$. Recall that an Arthur parameter, or an $A$-parameter, for $G$ is a continuous homomorphism 
$\psi: \mathcal{L}_{F} \times \mathrm{SL}(2, \mathbb{C}) \rightarrow G^{\vee}$ such that

- the restriction $\left.\psi\right|_{\mathcal{W}_{F}}$ of $\psi$ to the Weil group $\mathcal{W}_{F}$ is bounded,

- the image of $\left.\psi\right|_{\mathcal{W}_{F}}$ consists of semisimple elements of $G^{\vee}$,

- and the restriction of $\psi$ to each of the two $\operatorname{SL}(2, \mathbb{C})$ factors is algebraic.

A Langlands parameter, or an $L$-parameter, for $G$ is a continuous homomorphism $\phi: \mathcal{L}_{F} \rightarrow G^{\vee}$ such that

- the image of $\left.\phi\right|_{\mathcal{W}_{F}}$ consists of semisimple elements of $G^{\vee}$,

- and the restriction of $\phi$ to $\mathrm{SL}(2, \mathbb{C})$ is algebraic.

Inspired by work of Gaitsgory and Nadler, Sakellaridis and Venkatesh have associated to any $\mathbf{G}$-spherical $F$-variety $\mathbf{X}$ a complex dual group $G_{X}^{\vee}$, see [SV17, Sections 2-3] (provided that the assumption of [SV17, Proposition 2.2.2] on the spherical roots is satisfied). In addition, they described a distinguished morphism $\varrho$ : $G_{X}^{\vee} \times \mathrm{SL}(2, \mathbb{C}) \rightarrow G^{\vee}$ satisfying certain properties and unique up to conjugation by a canonical maximal torus in $G^{\vee}$ [SV17, Section 3.2]. Existence of distinguished morphisms has been proven in full generality by Knop and Schalke KS17.

Definition 4.1. An $A$-parameter $\psi: \mathcal{L}_{F} \times \mathrm{SL}(2, \mathbb{C}) \rightarrow G^{\vee}$ is $X$-distinguished if it factors through the distinguished morphism $\varrho: G_{X}^{\vee} \times \operatorname{SL}(2, \mathbb{C}) \rightarrow G^{\vee}$, that is, if and only if there exists a tempered (that is, bounded on $\mathcal{W}_{F}$ ) $L$-parameter $\phi_{X}: \mathcal{L}_{F} \rightarrow G_{X}^{\vee}$ such that $\psi=\varrho \circ\left(\phi_{X} \times \operatorname{Id}\right)$, where $\operatorname{Id}: \operatorname{SL}(2, \mathbb{C}) \rightarrow \operatorname{SL}(2, \mathbb{C})$ is the identity.

Definition 4.2. An $X$-distinguished $A$-parameter is $X$-elliptic if it factors through $\varrho$ via an elliptic $L$-parameter $\phi_{X}: \mathcal{L}_{F} \rightarrow G_{X}^{\vee}$, that is, the image of $\phi_{X}$ is not contained in any proper Levi subgroup of $G_{X}^{\vee}$.

We recall the following conjecture [SV17, Conjecture 1.3.1].

Conjecture 4.3 (Sakellaridis-Venkatesh). The support of the Plancherel measure for $L^{2}(X)$, as a representation of $G$, is contained in the union of Arthur packets attached to $X$-distinguished $A$-parameters.

In fact, Sakellaridis and Venkatesh give much more refined conjectures that predict a direct integral decomposition of $L^{2}(X)$ over $X$-distinguished $A$-parameters [SV17, Conjecture 16.2.2]. In addition, the refined conjectures make the following prediction about the $X$-distinguished $A$-parameters of the relative discrete series representations.

Conjecture 4.4 (Sakellaridis-Venkatesh). A relative discrete series representation $\pi$ in $L^{2}(X)$ is contained in an Arthur packet corresponding to an $X$-distinguished $X$-elliptic A-parameter.

Of course, in the setting we are concerned with, the situation is greatly simplified by the fact that Arthur packets (and $L$-packets) for the general linear group are singleton sets.

4.1. Distinguished $A$-parameters for $\mathbf{S p}_{2 n} \backslash \mathbf{G} \mathbf{L}_{2 n}$. Let $\mathbf{X}$ be the symmetric variety $\mathbf{S p}_{2 n} \backslash \mathbf{G L}_{2 n}$. The dual group of $G=\mathbf{G L}_{2 n}(F)$ is $G^{\vee}=\operatorname{GL}(2 n, \mathbb{C})$. The dual group $G_{X}^{\vee}$ of $\mathbf{X}$ is the rank- $n$ complex general linear group, that is, $G_{X}^{\vee}=\operatorname{GL}(n, \mathbb{C})$ (see Lemma 5.6).

Let $\pi$ be an irreducible unitary $\mathbf{S p}_{2 n}(F)$-distinguished representation of $G$. Let $\psi_{\pi}: \mathcal{L}_{F} \times \mathrm{SL}(2, \mathbb{C}) \rightarrow \mathrm{GL}(2 n, \mathbb{C})$ be the $A$-parameter of $\pi$. We refer the reader to 
Art13, Xu17, for the description of the $A$-parameters of the representations in the unitary dual of $G$, including the generalized Steinberg and Speh representations. The distinguished morphism $\varrho: \operatorname{GL}(n, \mathbb{C}) \times \mathrm{SL}(2, \mathbb{C}) \rightarrow \mathrm{GL}(2 n, \mathbb{C})$ is given by the tensor product of the standard $n$-dimensional representation of $\mathrm{GL}(n, \mathbb{C})$ with the standard 2-dimensional representation $\mathcal{S}(2)$ of $\mathrm{SL}(2, \mathbb{C})$ [SV17, Example 1.3.2]. Thus, if $\phi: \mathcal{L}_{F} \rightarrow \operatorname{GL}(n, \mathbb{C})$ is an $L$-parameter, then $\varrho \circ(\phi \times \mathrm{Id})=\phi \otimes \mathcal{S}(2)$. By Conjecture 4.4, we expect $\pi$ to be relatively square integrable if its $A$-parameter $\psi_{\pi}$ has the following two properties:

P1: The $A$-parameter $\psi_{\pi}$ is $X$-distinguished, that is, $\psi_{\pi}$ factors through the distinguished morphism $\varrho$; in particular, $\psi_{\pi}=\phi_{\pi, X} \otimes \mathcal{S}(2)$, where $\phi_{\pi, X}$ : $\mathcal{L}_{F} \rightarrow \mathrm{GL}(n, \mathbb{C})$ is a tempered $L$-parameter.

P2: The $L$-parameter $\phi_{\pi, X}: \mathcal{L}_{F} \rightarrow \mathrm{GL}(n, \mathbb{C})$ is elliptic, that is, the image of $\phi_{\pi, X}$ is not contained in any proper parabolic subgroup of $\mathrm{GL}(n, \mathbb{C})$.

Recall that, under the Local Langlands Correspondence, a tempered elliptic parameter $\phi: \mathcal{L}_{F} \rightarrow \mathrm{GL}(n, \mathbb{C})$ corresponds to a discrete series representation of $\mathbf{G L}_{n}(F)$.

Proposition 4.5. Let $\pi$ be an irreducible unitary $\mathbf{S p}_{2 n}(F)$-distinguished representation of $\mathbf{G L}_{2 n}(F)$. Let $\psi_{\pi}: \mathcal{L}_{F} \times \mathrm{SL}(2, \mathbb{C}) \rightarrow \mathrm{GL}(2 n, \mathbb{C})$ be the A-parameter of $\pi$. The A-parameter $\psi_{\pi}$ is $X$-distinguished and $X$-elliptic if and only if $\pi$ is isomorphic to a Speh representation $\mathcal{U}(\delta, 2)$ for some discrete series representation $\delta$ of $\mathbf{G L}_{n}(F)$.

Proof. Let $\pi$ be an irreducible unitary $\mathbf{S p}_{2 n}(F)$-distinguished representation of $\mathbf{G L}_{2 n}(F)$. Following the notation of OS07, let $\pi(\sigma, \alpha)=\nu^{\alpha} \sigma \times \nu^{-\alpha} \sigma$, where $\alpha \in \mathbb{R}$ so that $|\alpha|<1 / 2$, and $\sigma$ is a smooth representation of $\mathbf{G L}_{d}(F)$, for some $d \geq 1$. Offen and Sayag OS07, OS08b] have shown that $\pi$ must be equivalent to a representation of the form

$$
\left(\underset{i=1}{\operatorname{l}} \mathcal{U}\left(\mathcal{Z}\left(\rho_{i}, k_{i}\right), 2 m_{i}\right)\right) \times\left(\underset{i=l+1}{\underset{x}{X}} \pi\left(\mathcal{U}\left(\mathcal{Z}\left(\rho_{i}, k_{i}\right), 2 m_{i}\right), \alpha_{i}\right)\right),
$$

where $2 n=\sum_{i=1}^{l} 2 k_{i} r_{i} m_{i}+\sum_{i=l+1}^{t} 4 k_{i} r_{i} m_{i}$, the representations $\rho_{i}$ of $\mathbf{G L}_{r_{i}}(F)$ are irreducible, unitary and supercuspidal, and $\alpha_{i} \in \mathbb{R}$ with $\left|\alpha_{i}\right|<1 / 2$.

Let $\phi_{\rho_{i}}: \mathcal{W}_{F} \rightarrow \mathrm{GL}\left(r_{i}, \mathbb{C}\right)$ be the $L$-parameter of the supercuspidal representation $\rho_{i}$. Write $\mathcal{S}(d) \cong \operatorname{Sym}^{d-1}\left(\mathbb{C}^{2}\right)$ for the unique (up to isomorphism) $d$ dimensional irreducible representation of $\mathrm{SL}(2, \mathbb{C})$. Let $|\cdot| \mathcal{W}_{F}: \mathcal{W}_{F} \rightarrow \mathbb{R}_{>0}$ denote the absolute value on the Weil group given by $|\cdot| \mathcal{W}_{F}=\left|\operatorname{Art}_{F}^{-1}(\cdot)\right|_{F}$, where $\operatorname{Art}_{F}: F^{\times} \rightarrow \mathcal{W}_{F}^{\text {ab }}$ is the Artin map and $|\cdot|_{F}$ is the (normalized) absolute value on $F$. Up to equivalence, the $A$-parameter $\psi_{\pi}$ of $\pi$ is equal to

$$
\bigoplus_{i=1}^{l} \psi_{\mathcal{U}\left(\mathcal{Z}\left(\rho_{i}, k_{i}\right), 2 m_{i}\right)} \oplus \bigoplus_{i=l+1}^{t}\left(\left.|\cdot|_{\mathcal{W}_{F}}^{\alpha_{i}} \psi_{\mathcal{U}\left(\mathcal{Z}\left(\rho_{i}, k_{i}\right), 2 m_{i}\right)} \oplus|\cdot|\right|_{\mathcal{W}_{F}} ^{-\alpha_{i}} \psi_{\mathcal{U}\left(\mathcal{Z}\left(\rho_{i}, k_{i}\right), 2 m_{i}\right)}\right)
$$

where

$$
\psi_{\mathcal{U}\left(\mathcal{Z}\left(\rho_{i}, k_{i}\right), 2 m_{i}\right)}=\phi_{\mathcal{Z}\left(\rho_{i}, k_{i}\right)} \otimes \mathcal{S}\left(2 m_{i}\right): \mathcal{L}_{F} \times \mathrm{SL}(2, \mathbb{C}) \rightarrow \mathrm{GL}\left(2 k_{i} r_{i} m_{i}, \mathbb{C}\right)
$$

is the $A$-parameter of the generalized Speh representation $\mathcal{U}\left(\mathcal{Z}\left(\rho_{i}, k_{i}\right), 2 m_{i}\right)$, and

$$
\phi_{\mathcal{Z}\left(\rho_{i}, k_{i}\right)}=\phi_{\rho_{i}} \otimes \mathcal{S}\left(k_{i}\right): \mathcal{L}_{F} \rightarrow \mathrm{GL}\left(k_{i} r_{i}, \mathbb{C}\right)
$$

is the $L$-parameter of the generalized Steinberg representation $\mathcal{Z}\left(\rho_{i}, k_{i}\right)$. 
The $A$-parameter $\psi_{\pi}$ is $X$-distinguished $(\mathbf{P 1})$ if and only if $\psi_{\pi}=\phi_{\pi, X} \otimes \mathcal{S}(2)$, for some tempered $L$-parameter $\phi_{\pi, X}: \mathcal{L}_{F} \rightarrow \operatorname{GL}(n, \mathbb{C})$. In light of (4.1) and (4.2), we first notice that $\psi_{\pi}$ factors through the distinguished morphism $\varrho$ if and only if $m_{i}=1$, for all $1 \leq i \leq t$. In this case,

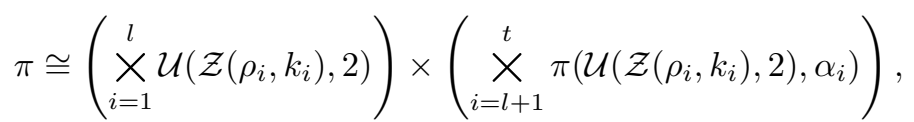

and

$$
\begin{aligned}
& \psi_{\pi}=\bigoplus_{i=1}^{l} \psi_{\mathcal{U}\left(\mathcal{Z}\left(\rho_{i}, k_{i}\right), 2\right)} \oplus \bigoplus_{i=l+1}^{t}\left(\left.|\cdot|_{\mathcal{W}_{F}}^{\alpha_{i}} \psi_{\mathcal{U}\left(\mathcal{Z}\left(\rho_{i}, k_{i}\right), 2\right)} \oplus|\cdot|\right|_{\mathcal{W}_{F}} ^{-\alpha_{i}} \psi_{\mathcal{U}\left(\mathcal{Z}\left(\rho_{i}, k_{i}\right), 2\right)}\right) \\
& =\left(\left.\bigoplus_{i=1}^{l} \phi_{\mathcal{Z}\left(\rho_{i}, k_{i}\right)} \oplus \bigoplus_{i=l+1}^{t}|\cdot|_{\mathcal{W}_{F}}^{\alpha_{i}} \phi_{\mathcal{Z}\left(\rho_{i}, k_{i}\right)} \oplus|\cdot|\right|_{\mathcal{W}_{F}} ^{-\alpha_{i}} \phi_{\mathcal{Z}\left(\rho_{i}, k_{i}\right)}\right) \otimes \mathcal{S}(2) \\
& =\phi_{\pi, X} \otimes \mathcal{S}(2),
\end{aligned}
$$

where $\phi_{\pi, X}: \mathcal{L}_{F} \rightarrow \mathrm{GL}(n, \mathbb{C})$ is the $L$-parameter

$$
\left.\bigoplus_{i=1}^{l} \phi_{\mathcal{Z}\left(\rho_{i}, k_{i}\right)} \oplus \bigoplus_{i=l+1}^{t}|\cdot|_{\mathcal{W}_{F}}^{\alpha_{i}} \phi_{\mathcal{Z}\left(\rho_{i}, k_{i}\right)} \oplus|\cdot|\right|_{\mathcal{W}_{F}} ^{-\alpha_{i}} \phi_{\mathcal{Z}\left(\rho_{i}, k_{i}\right)} .
$$

Moreover, the $L$-parameter $\phi_{\pi, X}$ is tempered if and only if $\phi_{\pi, X}$ restricted to $\mathcal{W}_{F}$ has bounded image, that is, if and only if $l=t$ (or, equivalently, $\alpha_{i}=0$ for all $l+1 \leq i \leq t)$. In particular, the representations $\pi\left(\mathcal{U}\left(\mathcal{Z}\left(\rho_{i}, k_{i}\right), 2\right), \alpha_{i}\right)$ cannot appear in the inducing data of $\pi$. Thus, $\psi_{\pi}$ is $X$-distinguished (P1) if and only if $\psi_{\pi}=\phi_{\pi, X} \otimes \mathcal{S}(2)$, where

$$
\phi_{\pi, X}=\bigoplus_{i=1}^{l} \phi_{\mathcal{Z}\left(\rho_{i}, k_{i}\right)} .
$$

The $L$-parameter $\phi_{\pi, X}$ is $X$-elliptic (P2) if and only if there is precisely one direct summand in (4.3) (that is, $l=1$ ) and $\phi_{\pi, X}=\phi_{\mathcal{Z}(\rho, k)}$ corresponds to the discrete series representation $\delta=\mathcal{Z}(\rho, k)$ of $\mathbf{G L}_{n}(F)$. In particular, $\psi_{\pi}$ is $X$-distinguished (P1) and $X$-elliptic (P2) if and only if $\psi_{\pi}=\phi_{\mathcal{Z}(\rho, k)} \otimes \mathcal{S}(2)$, in which case, by the Local Langlands Correspondence, $\pi \cong \mathcal{U}(\delta, 2)$ is a Speh representation.

In summary, Proposition 4.5 allows us to interpret Conjecture 4.4 as predicting that only the Speh representations $\mathcal{U}(\delta, 2)$, where $\delta=\mathcal{Z}(\rho, k)$ is a discrete series representation of $\mathbf{G} \mathbf{L}_{n}(F)$, appear in the discrete spectrum of $X=\mathbf{S p}_{2 n}(F) \backslash \mathbf{G L}_{2 n}(F)$. The goal of the rest of this paper is to prove that the representations $\mathcal{U}(\delta, 2)$ do indeed appear in $L_{\text {disc }}^{2}(X)$.

Remark 4.6. We do not show that generalized Speh representations $\mathcal{U}(\delta, 2 m), m \geq$ 2 , are not relatively square integrable despite the fact that Conjecture 4.4 predicts that these representations do not appear in $L_{\text {disc }}^{2}(X)$. See [Smi18b, Remark 6.6] for a discussion of the difficulties therein.

\section{Tori and Parabolic subgroups: Structure of $\mathbf{S p}_{2 n}(F) \backslash \mathbf{G L}_{2 n}(F)$}

In this section, we identify the $\theta$-split parabolic subgroups required for our application of the Relative Casselman Criterion. First we introduce a second involution 
that is $G$-equivalent to $\theta$ ( $c f$. Section 2.4). Let $w_{+} \in G$ be the permutation matrix associated to the permutation

$$
\begin{cases}2 i-1 \mapsto i & 1 \leq i \leq n \\ 2 i \mapsto 2 n+1-i & 1 \leq i \leq n\end{cases}
$$

of $\{1, \ldots, 2 n\}$. We have chosen $w_{+}$such that

$$
\varepsilon_{2 n} \cdot w_{+}={ }^{t} w_{+} \varepsilon_{2 n} w_{+}=w_{+}^{-1} \varepsilon_{2 n} w_{+}=\left(\begin{array}{ccccc}
0 & 1 & & & \\
-1 & 0 & & & \\
& & \ddots & & \\
& & 0 & 1 \\
& & & -1 & 0
\end{array}\right) \text {. }
$$

Let $x_{2 n}$ denote the nonsingular skew-symmetric matrix $\varepsilon_{2 n} \cdot w_{+}$and let $\theta_{x_{2 n}}$ be the associated involution of $G$. As above, we have that $\theta_{x_{2 n}}=\theta_{\varepsilon_{2 n} \cdot w_{+}}=w_{+} \cdot \theta$, and $\theta_{x_{2 n}}$ is $G$-equivalent to $\theta$.

Lemma 5.1. Let $A_{0}$ be the maximal diagonal F-split torus of $G$. The torus $A_{0}$ is $\theta$-stable and contains the maximal $(\theta, F)$-split torus $S_{0}$, where

$$
S_{0}=\left\{\operatorname{diag}\left(a_{1}, \ldots, a_{n}, a_{n}, \ldots, a_{1}\right): a_{i} \in F^{\times}, 1 \leq i \leq n\right\} .
$$

Proof. Let $a=\operatorname{diag}\left(a_{1}, \ldots, a_{2 n}\right) \in A_{0}$. First note that

$$
\theta(a)=\operatorname{diag}\left(a_{2 n}^{-1}, \ldots, a_{1}^{-1}\right) .
$$

In particular, $a$ is $\theta$-split if and only if $a_{2 n+1-i}=a_{i}$ for all $1 \leq i \leq n$. The torus $S_{0}$ is the $(\theta, F)$-split component of $A_{0}$. Thus, it is sufficient to show that $S_{0}$ is a maximal $(\theta, F)$-split torus in $G$. To do so, we will prove that the block-upper triangular parabolic $P_{(\underline{2})}$ corresponding to the partition $(\underline{2})=(2, \ldots, 2)$ of $2 n$ is a minimal $\theta_{x_{2 n}}$-split parabolic, and then use the $G$-equivalence of $\theta_{x_{2 n}}$ and $\theta$ to conclude that $P_{0}=w_{+} P_{(2)} w_{+}^{-1}$ is a minimal $\theta$-split parabolic subgroup of $G$. The desired result then follows from [HW93, Proposition 4.7(iv)].

To see that $P_{(\underline{2})}$ is $\theta_{x_{2 n}}$-split, first note that $x_{2 n} \in M_{(\underline{2})}$; therefore, the blockdiagonal Levi $M_{(\underline{2})}$ is $\theta_{x_{2 n}}$-stable. The unipotent radical $N_{(\underline{2})}$ of $P_{(\underline{2})}$ is mapped to the opposite unipotent radical $N_{(2)}^{\text {op }}$ (with respect to $M_{(\underline{2})}$ ) by taking conjugatetranspose, and both $N_{(\underline{2})}$ and $N_{(\underline{2})}^{\mathrm{op}}$ are normalized by $M_{(\underline{2})}$. It follows that $\theta_{x_{2 n}}\left(P_{(\underline{2})}\right)$ $=M_{(\underline{2})} N_{(\underline{2})}^{\mathrm{op}}=P_{(\underline{2})}^{\mathrm{op}}$ and $P_{(\underline{2})}$ is $\theta_{x_{2 n}}$-split. It only remains to show that $P_{(\underline{2})}$ is a minimal $\theta_{x_{2 n}}$-split parabolic subgroup. Suppose that $P=M N \subsetneq P_{(\underline{2})}$ is a $\theta_{x_{2 n}}$ split parabolic subgroup of $G$ that is properly contained in $P_{(\underline{2})}$. The parabolic subgroup $P \cap M_{(\underline{2})}$ of $M_{(\underline{2})}$ is $\theta_{x_{2 n}}$-split in $M_{(\underline{2})}$. Notice that the GL-blocks of $M_{(\underline{2})}$ are not interchanged by $\theta_{x_{2 n}}$. In fact, $\theta_{x_{2 n}}$ restricted to $M_{(\underline{2})}$ is equal to the product $\theta_{x_{2}} \times \ldots \times \theta_{x_{2}}$. It follows that $P \cap M_{(\underline{2})}$ is a product of $\theta x_{2}$-split parabolic subgroups in $\mathbf{G L}_{2}(F)$. Notice that the $F$-split component of the centre of $M_{(\underline{2})}$ is $\left(\theta_{x_{2 n}}, F\right)$-split. By [HW93, Proposition 4.7(iv)], no proper parabolic subgroup of $\mathbf{G L}_{2}(F)$ can be $\theta x_{2}$-split, and it follows that $M_{(\underline{2})}$ has no proper $\theta_{x_{2 n}}$-split parabolic subgroups. In particular, $P_{(\underline{2})}$ is a minimal $\theta_{x_{2 n}}$-split parabolic subgroup of $G$.

The torus $S_{0, x_{2 n}}=\left\{\operatorname{diag}\left(a_{1}, a_{1}, \ldots, a_{n}, a_{n}\right): a_{i} \in F^{\times}\right\}$is a maximal $\left(\theta_{x_{2 n}}, F\right)$ split torus of $G$, it is the $\left(\theta_{x_{2 n}}, F\right)$-split component of $P_{(\underline{2})}$ and the $F$-split component 
of $M_{(\underline{2})}$. The torus $S_{0}$ is the $w_{+}$-conjugate of $S_{0, x_{2 n}}$. We also note explicitly that $P_{0}=w_{+} P_{(2)} w_{+}^{-1}$ is $\theta$-split:

$$
\begin{aligned}
\theta\left(P_{0}\right) & =\theta\left(w_{+} P_{(\underline{2})} w_{+}^{-1}\right) \\
& =w_{+} w_{+}^{-1} \theta\left(w_{+} P_{(\underline{2})} w_{+}^{-1}\right) w_{+} w_{+}^{-1} \\
& =w_{+} \theta_{x_{2 n}}\left(P_{(\underline{2})}\right) w_{+}^{-1} \\
& =w_{+}\left(P_{(\underline{2})}^{\mathrm{op}}\right) w_{+}^{-1} \\
& =P_{0}^{\mathrm{op}}
\end{aligned}
$$

where the opposite is taken with respect to the $\theta$-stable Levi factor $M_{0}=w_{+} M_{(\underline{2})} w_{+}^{-1}$. Let $N_{0}=w_{+} N_{(\underline{2})} w_{+}^{-1}$ denote the unipotent radical of $P_{0}$. We emphasize that $P_{0}=M_{0} N_{0}$ is a minimal $\theta$-split parabolic subgroup of $G$.

\subsection{The restricted root system and $\theta$-split parabolic subgroups.}

Definition 5.2. Let $\Delta$ be a base of a root system $\Phi$. The $\Delta$-positive (respectively, $\Delta$-negative) roots in $\Phi$ consist of the collection of positive (respectively, negative) roots in $\Phi$ with respect to $\Delta$; in particular, the set of $\Delta$-positive roots is equal to $\Phi \cap \operatorname{span}_{\mathbb{Z}_{>0}} \Delta$.

Let $\Phi_{0}=\Phi\left(\mathbf{G}, \mathbf{A}_{0}\right)$ be the root system of $G$ with respect to $A_{0}$. Since $A_{0}$ is $\theta$-stable, the involution $\theta$ acts on $X^{*}\left(A_{0}\right)$ and $\Phi_{0}$ is $\theta$-stable under this action. Let $\Delta=\left\{\epsilon_{i}-\epsilon_{i+1}: 1 \leq i \leq 2 n-1\right\}$ be the standard base for $\Phi_{0}$, where $\epsilon_{i}$ denotes the $i$-th $F$-rational coordinate character of $A_{0}$. Define $\Delta_{0}=w_{+} \Delta$ to be the Weyl group translate of $\Delta$ by the permutation matrix $w_{+} \in W_{0}$, where $W_{0} \cong N_{G}\left(A_{0}\right) / A_{0}$ is the Weyl group of $G$ (with respect to $A_{0}$ ). We identify $W_{0}$ with the subgroup of $G$ consisting of all permutation matrices.

Lemma 5.3. The set $\Phi_{0}^{\theta}$ of $\theta$-fixed roots in $\Phi_{0}$ is equal to the set

$$
\Phi_{0}^{\theta}=\left\{\epsilon_{i}-\epsilon_{2 n+1-i}: 1 \leq i \leq 2 n\right\},
$$

corresponding to the root spaces on the main anti-diagonal in $\mathfrak{g l}_{2 n}$.

Proof. For any $1 \leq i \neq j \leq 2 n$, we have that $\theta\left(\epsilon_{i}-\epsilon_{j}\right)=\epsilon_{2 n+1-j}-\epsilon_{2 n+1-i}$. Note that $2 n+1-(2 n+1-i)=i$; therefore, the root $\epsilon_{i}-\epsilon_{j}$ is $\theta$-fixed if and only if $j=2 n+1-i$.

Lemma 5.4. The set of simple roots $\Delta_{0}=w_{+} \Delta$ is a $\theta$-base of $\Phi_{0}$.

Proof. The set $\Phi_{0}^{+}$of $\Delta_{0}$-positive roots is equal to $w_{+} \Phi_{\Delta}^{+}$, where $\Phi_{\Delta}^{+}$is the set of $\Delta$ positive roots. Moreover, the set of $\Delta_{0}$-negative roots in $\Phi_{0}$ is $\Phi_{0}^{-}=-\Phi_{0}^{+}=w_{+} \Phi_{\Delta}^{-}$. Let $\alpha=\epsilon_{i}-\epsilon_{j} \in \Phi_{\Delta}^{+}$, that is, $1 \leq i<j \leq 2 n$ and $w_{+} \alpha \in \Phi_{0}^{+}$. Suppose that $w_{+} \alpha$ is not $\theta$-fixed. Note that $w_{+} \epsilon_{i}=\epsilon_{w_{+}(i)}$ and thus

$$
\theta\left(w_{+}\left(\epsilon_{i}-\epsilon_{j}\right)\right)=\epsilon_{2 n+1-w_{+}(j)}-\epsilon_{2 n+1-w_{+}(i)} .
$$

We consider the image of $w_{+} \alpha$ under $\theta$ in the following four cases.

Case (i): $i, j$ both odd. We can write $i=2 k-1$ and $j=2 l-1$ with $1 \leq k<l \leq n$. It follows that

$$
\theta\left(w_{+} \alpha\right)=\epsilon_{2 n+1-l}-\epsilon_{2 n+1-k}=w_{+}\left(\epsilon_{2 l}-\epsilon_{2 k}\right) ;
$$

moreover, since $2 l>2 k$, we have that $w_{+}\left(\epsilon_{2 l}-\epsilon_{2 k}\right) \in \Phi_{0}^{-}$. 
Case (ii): $i$ odd, $j$ even. Let $i=2 k-1$ and $j=2 l$ with $1 \leq k \leq l \leq n$. As above,

$$
\theta\left(w_{+} \alpha\right)=\epsilon_{l}-\epsilon_{2 n+1-k}=w_{+}\left(\epsilon_{2 l-1}-\epsilon_{2 k}\right) .
$$

Observe that $k \neq l$, since otherwise $w_{+} \alpha=\theta\left(w_{+} \alpha\right) \in \Phi_{0}^{\theta}$ and we have assumed that $w_{+} \alpha$ is not $\theta$-fixed. Since $l>k$, we have $2 l-1>2 k$ and $w_{+}\left(\epsilon_{2 l}-\epsilon_{2 k}\right) \in \Phi_{0}^{-}$. Case (iii): $i$ even, $j$ odd. Let $i=2 k$ and $j=2 l-1$ where $1 \leq k<l \leq n$. It follows that

$$
\theta\left(w_{+} \alpha\right)=\epsilon_{2 n+1-l}-\epsilon_{2 n+1-(2 n+1-k)}=w_{+}\left(\epsilon_{2 l}-\epsilon_{2 k-1}\right) ;
$$

moreover, since $l>k$, we have $2 l>2 k-1$ and $w_{+}\left(\epsilon_{2 l}-\epsilon_{2 k-1}\right) \in \Phi_{0}^{-}$.

Case (iv): $i, j$ both even. Let $i=2 k$ and $j=2 l$ for $1 \leq k<l \leq n$. We have

$$
\theta\left(w_{+}\left(\epsilon_{2 k}-\epsilon_{2 l}\right)\right)=\epsilon_{2 n+1-(2 n+1-l)}-\epsilon_{2 n+1-(2 n+1-k)}=w_{+}\left(\epsilon_{2 l-l}-\epsilon_{2 k-1}\right) ;
$$

moreover, since $l>k$, we have that $2 l-1>2 k-1$ and $w_{+}\left(\epsilon_{2 l-1}-\epsilon_{2 k-1}\right) \in \Phi_{0}^{-}$.

It follows that if $\beta \in \Phi_{0}^{+}$is not $\theta$-fixed, then $\theta(\beta) \in \Phi_{0}^{-}$; therefore, $\Delta_{0}$ is a $\theta$-base of $\Phi_{0}$.

Observation 5.5. From the proof of Lemma 5.4, we see that the set of $\theta$-fixed $\Delta_{0}$-positive roots are the translates of $\left\{\epsilon_{1}-\epsilon_{2}, \epsilon_{3}-\epsilon_{4}, \ldots, \epsilon_{2 n-1}-\epsilon_{2 n}\right\}$ by $w_{+}$. The subset $\left\{\epsilon_{1}-\epsilon_{2}, \epsilon_{3}-\epsilon_{4}, \ldots, \epsilon_{2 n-1}-\epsilon_{2 n}\right\}$ of $\Delta$ consists of $\theta_{x_{2 n}}$-fixed roots and determines the (minimal $\theta_{x_{2 n}}$-split) parabolic subgroup $P_{(\underline{2})}$.

To aid in our understanding of the structure of $\Delta_{0}$, we partition the roots in the standard base $\Delta$ into the disjoint subsets

$$
\Delta_{\text {odd }}=\left\{\epsilon_{2 i-1}-\epsilon_{2 i}: 1 \leq i \leq n\right\}
$$

and

$$
\Delta_{\text {even }}=\left\{\epsilon_{2 j}-\epsilon_{2 j+1}: 1 \leq j \leq n-1\right\} .
$$

Notice that the set of $\theta$-fixed simple roots in $\Delta_{0}$ is equal to $\Delta_{0}^{\theta}=w_{+} \Delta_{\text {odd }}$. Moreover, $\Delta_{0}$ is the disjoint union $\Delta_{0}=\Delta_{0}^{\theta} \sqcup w_{+} \Delta_{\text {even }}$. Explicitly,

$$
\Delta_{0}^{\theta}=w_{+} \Delta_{\text {odd }}=\left\{\epsilon_{i}-\epsilon_{2 n+1-i}: 1 \leq i \leq n\right\}
$$

and

$$
w_{+} \Delta_{\text {even }}=\left\{\epsilon_{2 n+1-j}-\epsilon_{j+1}: 1 \leq j \leq n-1\right\} .
$$

Let $r: X^{*}\left(A_{0}\right) \rightarrow X^{*}\left(S_{0}\right)$ be the surjective homomorphism defined by restricting $F$-rational characters of $A_{0}$ to $S_{0}$. The $\theta$-fixed simple roots are trivial on $S_{0}$. It follows that

$$
\bar{\Delta}_{0}=r\left(\Delta_{0} \backslash \Delta_{0}^{\theta}\right)=r\left(w_{+} \Delta_{\text {even }}\right)=\left\{\bar{\epsilon}_{i}-\bar{\epsilon}_{i+1}: 1 \leq i \leq n-1\right\},
$$

where $\bar{\epsilon}_{i}$ is the $i$-th $F$-rational coordinate character of $S_{0}$ given by

$$
\bar{\epsilon}_{i}\left(\operatorname{diag}\left(a_{1}, \ldots, a_{n}, a_{n}, \ldots, a_{1}\right)\right)=a_{i},
$$

for $1 \leq i \leq n$. In addition, the full set of restricted roots is

$$
\bar{\Phi}_{0}=r\left(\Phi_{0}\right) \backslash\{0\}=r\left(\Phi_{0} \backslash \Phi_{0}^{\theta}\right)=\left\{\bar{\epsilon}_{i}-\bar{\epsilon}_{j}: 1 \leq i \neq j \leq n\right\} .
$$

We have established the following.

Lemma 5.6. The restricted root system associated to $\mathbf{S p}_{2 n}(F) \backslash \mathbf{G L}_{2 n}(F)$ is of type $\mathrm{A}_{n-1}$ and the dual group $G_{X}^{\vee}$ of $X=\mathbf{S p}_{2 n}(F) \backslash \mathbf{G L}_{2 n}(F)$ is $\mathrm{GL}(n, \mathbb{C})$. 
Proper $\left(\Delta_{0^{-}}\right)$standard $\theta$-split parabolic subgroups of $G$ are parametrized by proper $\theta$-split subsets of $\Delta_{0}$, where a subset $\Theta$ of $\Delta_{0}$ is $\theta$-split if it is of the form

$$
\Theta=[\bar{\Theta}]:=r^{-1}(\bar{\Theta}) \cup \Delta_{0}^{\theta},
$$

and $\bar{\Theta}$ is a subset of $\bar{\Delta}_{0}$. The subset $\Delta_{0}^{\theta}$ of $\theta$-fixed simple roots determines the minimal standard $\theta$-split parabolic $P_{0}=M_{0} N_{0}$ of $G$, with Levi factor $M_{0}=C_{G}\left(S_{0}\right)$ and unipotent radical $N_{0}$. By [KT08, Lemma 2.5], any $\theta$-split parabolic subgroup of $G$ is $\left(\mathbf{H M}_{0}\right)(F)$-conjugate to a standard $\theta$-split parabolic. In the current setting, the Galois cohomology of $\mathbf{M}_{0} \cap \mathbf{H}$ over $F$ is trivial and it follows that $\left(\mathbf{H M}_{0}\right)(F)=$ $H M_{0}$; moreover, any $\theta$-split parabolic subgroup is $H$-conjugate to a standard $\theta$-split parabolic subgroup. For completeness, we give a proof.

Lemma 5.7. The first Galois cohomology of $\mathbf{M}_{0} \cap \mathbf{H}$ over $F$ is trivial and $\left(\mathbf{H M}_{0}\right)(F)=H M_{0}$.

Proof. First, one may readily verify that

$$
\mathbf{M}_{0} \cap \mathbf{H}=w_{+}\left(\mathbf{M}_{(\underline{2})} \cap \mathbf{G}^{\theta_{x_{2 n}}}\right) w_{+}^{-1} \cong \prod_{1}^{n} \mathbf{S L}_{2} .
$$

By Hilbert's Theorem 90, it follows that

$$
H^{1}\left(\mathbf{M}_{0} \cap \mathbf{H}, F\right) \cong \bigoplus_{1}^{n} H^{1}\left(\mathbf{S L}_{2}, F\right)=0 .
$$

Let $\bar{F}$ denote the algebraic closure of $F$. By considering the long exact sequence in Galois cohomology obtained from the short exact sequence

$$
1 \rightarrow \mathbf{M}_{0}(\bar{F}) \cap \mathbf{H}(\bar{F}) \rightarrow \mathbf{H}(\bar{F}) \times \mathbf{M}_{0}(\bar{F}) \rightarrow \mathbf{H}(\bar{F}) \mathbf{M}_{0}(\bar{F}) \rightarrow 1
$$

of pointed sets, it follows that $\left(\mathbf{H M}_{0}\right)(F)=H M_{0}$, as claimed.

Proposition 5.8. Let $P$ be a $\theta$-split parabolic subgroup of $G$. There exists a $\theta$-split subset $\Theta$ of $\Delta_{0}$ and an element $h \in H$ such that $P=h P_{\Theta} h^{-1}$. Moreover, $P$ has unipotent radical $N=h N_{\Theta} h^{-1}$ and $\theta$-stable Levi factor $M=h M_{\Theta} h^{-1}$.

Proof. Apply Lemma 5.7 and [KT08, Lemma 2.5].

With the last result in hand, we explicitly determine the maximal proper standard $\theta$-split parabolic subgroups of $G$ which correspond to the maximal proper $\theta$ split subsets of $\Delta_{0}$. A maximal proper $\theta$-split subset of $\Delta_{0}$ has the form $\left[\bar{\Delta}_{0} \backslash\{\bar{\alpha}\}\right]=$ $r^{-1}\left(\bar{\Delta}_{0} \backslash\{\bar{\alpha}\}\right) \cup \Delta_{0}^{\theta}$, where $\bar{\alpha} \in \bar{\Delta}_{0}$. Observe that for each $\bar{\alpha} \in \bar{\Delta}_{0}$ there is a unique $\alpha \in w_{+} \Delta_{\text {even }}$ such that $r(\alpha)=\bar{\alpha}$. Precisely, the pre-image of $\bar{\epsilon}_{i}-\bar{\epsilon}_{i+1}$ under the restriction map $r: X^{*}\left(A_{0}\right) \rightarrow X^{*}\left(S_{0}\right)$ is $r^{-1}\left(\bar{\epsilon}_{i}-\bar{\epsilon}_{i+1}\right)=w_{+}\left(\epsilon_{2 i}-\epsilon_{2 i+1}\right)$, for each $1 \leq i \leq n-1$. It follows that for each $1 \leq k \leq n-1$ we have a maximal $\theta$-split subset of $\Delta_{0}$ given by

$$
\begin{aligned}
\Theta_{k} & =r^{-1}\left(\bar{\Delta}_{0} \backslash\left\{\bar{\epsilon}_{k}-\bar{\epsilon}_{k+1}\right\}\right) \cup \Delta_{0}^{\theta} \\
& =w_{+}\left(\Delta \backslash\left\{\epsilon_{2 k}-\epsilon_{2 k+1}\right\}\right) \\
& =\Delta_{0} \backslash\left\{\epsilon_{2 n+1-k}-\epsilon_{k+1}\right\} .
\end{aligned}
$$

To each $\Theta_{k}, 1 \leq k \leq n-1$, we associate the maximal $\Delta_{0}$-standard $\theta$-split parabolic subgroup

$$
P_{\Theta_{k}}=w_{+} P_{(2 k, 2 n-2 k)} w_{+}^{-1},
$$


with $\theta$-stable Levi factor $M_{\Theta_{k}}=w_{+} M_{(2 k, 2 n-2 k)} w_{+}^{-1}$ and unipotent radical $N_{\Theta_{k}}=$ $w_{+} N_{(2 k, 2 n-2 k)} w_{+}^{-1}$. Notice that $P_{\Theta_{k}}$ does indeed contain the minimal standard $\theta-$ split parabolic subgroup $P_{0}=w_{+} P_{(\underline{2})} w_{+}^{-1}$, corresponding to $\Delta_{0}^{\theta}$ (or the partition $(\underline{2})=(2, \ldots, 2)$ of $2 n)$. Moreover, by Lemma 5.1, the $(\theta, F)$-split component $S_{\Theta_{k}}$ of $P_{\Theta_{k}}$ is equal to its $F$-split component $A_{\Theta_{k}}$.

Note. It may be helpful to observe that the maximal $\theta_{x_{2 n}}$-split subsets of $\Delta$ are thus given by $\Delta \backslash\left\{\epsilon_{2 k}-\epsilon_{2 k+1}\right\}$, where $1 \leq k \leq n-1$. It follows that the standard block-upper-triangular parabolic subgroups $P_{(2 k, 2 n-2 k)}$, with even sized blocks, are the maximal $\Delta$-standard $\theta_{x_{2 n}}$-split parabolic subgroups.

\subsection{Inducing from distinguished representations of $\theta$-elliptic Levi sub-} groups. We recall the following definition.

Definition 5.9. A $\theta$-stable Levi subgroup of $G$ is $\theta$-elliptic if $L$ is not contained in any proper $\theta$-split parabolic subgroup of $G$.

In order to place the Speh representations within the context of the relative discrete series constructed in Smi18b, Smi18a, we show that $\mathcal{U}(\delta, 2)$ can be realized as the quotient of a representation induced from a distinguished representation of a $\theta$-elliptic Levi subgroup.

Lemma 5.10. The block-upper triangular parabolic subgroup $P_{(n, n)}$, corresponding to $\Omega^{\mathrm{ell}}=\Delta \backslash\left\{\epsilon_{n}-\epsilon_{n-1}\right\} \subset \Delta$, is $\theta$-stable and the $\Delta$-standard block-diagonal Levi subgroup $M_{(n, n)}$ is $\theta$-elliptic.

Proof. First, it is clear that $P_{(n, n)}$ and $M_{(n, n)}$ are $\theta$-stable subgroups of $G$. It is readily verified that the $(\theta, F)$-split component of $M_{(n, n)}$ is equal to the $(\theta, F)$-split component $S_{G}$ of $G$; moreover, $S_{G}=A_{G}$, that is, the $(\theta, F)$-split component of $G$ is equal to the $F$-split component of $G$. By [Smi18b, Lemma 3.8], the $\theta$-stable Levi subgroup $M_{(n, n)}$ is $\theta$-elliptic.

In what follows, we let $Q=P_{(n, n)}=P_{\Omega^{\text {ell }}}, L=M_{(n, n)}=M_{\Omega^{\text {ell }}}$, and $U=$ $N_{(n, n)}=N_{\Omega^{\text {ell }}}$. Define $\Omega=w_{+} \Omega^{\text {ell }} \subset \Delta_{0}$. We then have that $Q=w_{+}^{-1} P_{\Omega} w_{+}$or, equivalently, that $P_{\Omega}=w_{+} Q w_{+}^{-1}$.

Definition 5.11. An ordered partition $\left(m_{1}, \ldots, m_{k}\right)$ of an integer $m \geq 2$ is balanced if $\left(m_{1}, \ldots, m_{k}\right)$ is equal to the opposite partition $\left(m_{1}, \ldots, m_{k}\right)^{\mathrm{op}}=\left(m_{k}, \ldots\right.$, $\left.m_{1}\right)$.

Lemma 5.12. Let $P$ be a block-upper triangular ( $\Delta$-standard) parabolic subgroup of $G$. The subgroup $P$ is $\theta$-stable if and only if $P$ corresponds to a balanced partition of $2 n$. In addition, the only $\theta$-stable $\Delta$-standard maximal parabolic that admits a $\theta$-elliptic Levi subgroup is $P_{(n, n)}$.

Proof. The proof is the same as that of [Smi18a, Lemma 4.15].

Recall that a parabolic subgroup $P$ is $A_{0}$-semi-standard if $P$ contains the maximal $F$-split torus $A_{0}$. In particular, the $\Delta$ - and $\Delta_{0}$-standard parabolic subgroups are $A_{0}$-semi-standard. The next result is the analogue of [Smi18a, Lemma 4.21]; the proof is the same.

Lemma 5.13. Let $P$ be any $\theta$-stable parabolic subgroup of $G$. The subgroup $P$ is $H$-conjugate to a $\theta$-stable $A_{0}$-semi-standard parabolic subgroup. 
Lemma 5.14. The $\theta$-stable Levi subgroup $L=M_{(n, n)}$ is the only proper $\theta$-elliptic $A_{0}$-semi-standard Levi subgroup of $G$ up to conjugacy by Weyl group elements $w \in$ $W_{0}=W\left(G, A_{0}\right)=N_{G}\left(A_{0}\right) / A_{0}$ such that $w^{-1} \varepsilon_{2 n} w \in N_{G}(L) \backslash L$.

Proof. See the proof of [Smi18a, Lemma 4.20(2)].

Lemma 5.15. The group $L^{\theta}$ of $\theta$-fixed points in $L=M_{(n, n)}$ is isomorphic to $\mathbf{G L}_{n}(F)$ embedded in $L$ as follows:

$$
L^{\theta}=\left\{\left(\begin{array}{cc}
g & 0 \\
0 & J_{n}^{-1 t} g^{-1} J_{n}
\end{array}\right): g \in \mathbf{G L}_{n}(F)\right\} .
$$

Proof. We omit the straightforward calculation.

Proposition 5.16. Let $\tau_{1} \otimes \tau_{2}$ be an irreducible admissible representation of $L=$ $M_{(n, n)}$. Then $\tau_{1} \otimes \tau_{2}$ is $L^{\theta}$-distinguished if and only if $\tau_{2} \cong \tau_{1}$.

Proof. First, one can show that $\tau_{1} \otimes \tau_{2}$ is $L^{\theta}$-distinguished if and only if $\tau_{2} \cong$ $\widetilde{\tau}_{1} \circ \theta_{J_{n}}$, where $\theta_{J_{n}}$ is the involution on $\mathbf{G L}_{n}(F)$ given by $\theta_{J_{n}}(g)=J_{n}^{-1 t} g^{-1} J_{n}$, for $g \in \mathbf{G L}_{n}(F)$. Now, the lemma is a simple consequence of [GK75, Theorem 2] which implies that $\widetilde{\tau}_{1} \cong \tau_{1} \circ^{t}(\cdot)^{-1}$ and the fact that $J_{n}^{-1}=J_{n}={ }^{t} J_{n}$ (see [Smi18a, Lemma 5.3] for additional details).

Let $\tau$ be an irreducible admissible representation of $\mathbf{G} \mathbf{L}_{n}(F)$. The representation $\tau \otimes \tau$ of $L$ is $L^{\theta}$-distinguished by Proposition 5.16. Moreover, the $L^{\theta}$-invariant linear form on $\tau \otimes \tau$ can be realized via the standard pairing between $\tau$ and its contragredient $\widetilde{\tau}$. Indeed, this follows from [GK75, Theorem 2] and the fact that $\widetilde{\tau} \cong \tau \circ \theta_{J_{n}}$. Let $\lambda_{\tau} \in \operatorname{Hom}_{L^{\theta}}(\tau \otimes \tau, 1)$ be the (nonzero) invariant form that arises via the pairing on $\tau \otimes \widetilde{\tau}$. Let $l=\operatorname{diag}\left(x, \theta_{J_{n}}(x)\right) \in L^{\theta}$ and consider the value of $\delta_{Q^{\theta}} \delta_{Q}^{-1 / 2}$ on $l$. It is straightforward to check that

$$
\left(\left.\delta_{Q^{\theta}} \delta_{Q}^{-1 / 2}\right|_{L^{\theta}}\right)(l)=|\operatorname{det}(x)|^{n+1}|\operatorname{det}(x)|^{-n}=|\operatorname{det}(x)|=\nu(x),
$$

that is, $\delta_{Q^{\theta}} \delta_{Q}^{-1 / 2}$ agrees with the character $\nu$ on $\mathbf{G L}_{n}(F) \cong L^{\theta}$. Since the contragredient of $\nu$ is $\nu^{-1}$, it follows that $\lambda_{\tau} \in \operatorname{Hom}_{L^{\theta}}\left(\nu^{1 / 2} \tau \otimes \nu^{-1 / 2} \tau, \nu\right) \cong$ $\operatorname{Hom}_{L^{\theta}}\left(\delta_{Q}^{1 / 2} \tau \otimes \tau, \delta_{Q^{\theta}}\right)$. By [Off17, Proposition 7.1], $\lambda_{\tau}$ maps to a nonzero $H$ invariant linear form $\lambda \in \operatorname{Hom}_{H}\left(\nu^{1 / 2} \tau \times \nu^{-1 / 2} \tau, 1\right)$, and the parabolically induced representation $\nu^{1 / 2} \tau \times \nu^{-1 / 2} \tau=\iota_{Q}^{G}\left(\nu^{1 / 2} \tau \otimes \nu^{-1 / 2} \tau\right)$ is $H$-distinguished. We now state a result of Heumos and Rallis [HR90, Theorem 11.1] (cf. Section 3). We give a sketch of the proof (still appealing to the main results of [HR90]).

Proposition 5.17 (Heumos-Rallis). Let $\delta$ be an irreducible square integrable representation of $\mathbf{G L}_{n}(F)$. The parabolically induced representation $\nu^{1 / 2} \delta \times \nu^{-1 / 2} \delta=$ $\iota_{Q}^{G}\left(\nu^{1 / 2} \delta \otimes \nu^{-1 / 2} \delta\right)$ is $H$-distinguished. Moreover, the unique irreducible quotient $\mathcal{U}(\delta, 2)$ of $\nu^{1 / 2} \delta \times \nu^{-1 / 2} \delta$ is $H$-distinguished.

Proof. As above, $\nu^{1 / 2} \delta \times \nu^{-1 / 2} \delta$ is $H$-distinguished by Proposition 5.16 and Off17, Proposition 7.1]. The parabolically induced representation $\nu^{1 / 2} \delta \times \nu^{-1 / 2} \delta$ has length two [BZ77, Zel80]. Let $\mathcal{Z}(\delta, 2)$ be the unique irreducible subrepresentation and let $\mathcal{U}(\delta, 2)$ be the unique irreducible quotient of $\nu^{1 / 2} \delta \times \nu^{-1 / 2} \delta$. The subrepresentation $\mathcal{Z}(\delta, 2)$ is tempered and thus generic [Zel80, Theorem 9.3]. Therefore, by [HR90, Theorem 3.2.2], $\mathcal{Z}(\delta, 2)$ cannot be $H$-distinguished. It follows that any nonzero 
$H$-invariant linear form on $\nu^{1 / 2} \delta \times \nu^{-1 / 2} \delta$ descends to a well-defined nonzero $H$ invariant linear functional on the quotient $\mathcal{U}(\delta, 2)$.

Remark 5.18. By the multiplicity-one result HR90, Theorem 2.4.2], the $H$-invariant linear form on $\nu^{1 / 2} \tau \times \nu^{-1 / 2} \tau$ constructed via Off17. Proposition 7.1] is a scalar multiple of the invariant form produced by Heumos and Rallis in [HR90, §11.3.1.2] (cf. [Smi20, Lemma 1.3.4]).

\section{Application of the Relative Casselman Criterion}

We now come to the main result of the paper.

Theorem 6.1. Let $\delta$ be a discrete series representation of $\mathbf{G L}_{n}(F)$. The Speh representation $\mathcal{U}(\delta, 2)$ of $\mathbf{G L}_{2 n}(F)$ is $\mathbf{S p}_{2 n}(F)$-relatively square integrable.

Proof. Let $\lambda \in \operatorname{Hom}_{H}(\mathcal{U}(\delta, 2), 1)$ be nonzero. Let $\pi=\nu^{1 / 2} \delta \times \nu^{-1 / 2} \delta$. Recall from Section 3 that $\mathcal{U}(\delta, 2)$ is the unique irreducible quotient of $\pi$. By Proposition 5.8 and Smi18b, Proposition 4.22], it is enough to consider exponents along maximal standard $\theta$-split parabolic subgroups of $G$ when applying Theorem 2.7 (KT10, Theorem 4.7]). Let $P=M N$ be a maximal $\Delta_{0}$-standard $\theta$-split parabolic subgroup of $G$ with unipotent radical $N$ and $\theta$-stable Levi factor $M=P \cap \theta(P)$. By Smi18b, Proposition 4.23], only exponents corresponding to irreducible $M^{\theta}$-distinguished subquotients of the Jacquet module $\mathcal{U}(\delta, 2)_{N}$ may appear in $\operatorname{Exp}_{S_{M}}\left(\mathcal{U}(\delta, 2)_{N}, \lambda_{N}\right)$. By Proposition 6.2, the irreducible unitary subquotients of $\pi_{N}$, and also $\mathcal{U}(\delta, 2)_{N}$, are not $M^{\theta}$-distinguished. By Proposition 6.5, all exponents that appear in $E_{x p_{S_{M}}}\left(\mathcal{U}(\delta, 2)_{N}, \lambda_{N}\right)$ satisfy (2.2). By Theorem 2.7. $\mathcal{U}(\delta, 2)$ is $(H, \lambda)$-relatively square integrable. Multiplicity-one holds by [HR90, Theorem 2.4.2], thus dim $\mathrm{Hom}_{H}$ $(\mathcal{U}(\delta, 2), 1)=1$ and $\mathcal{U}(\delta, 2)$ is $H$-relatively square integrable.

The remainder of the paper is dedicated to proving Proposition 6.2 and Proposition 6.5

Let $\delta$ be an irreducible admissible square integrable (discrete series) representation of $\mathbf{G L}_{n}(F)$. Let $\pi=\nu^{1 / 2} \delta \times \nu^{-1 / 2} \delta$. The sequence

$$
0 \rightarrow \mathcal{Z}(\delta, 2) \rightarrow \pi \rightarrow \mathcal{U}(\delta, 2) \rightarrow 0
$$

of $G$-modules is exact, where $\mathcal{Z}(\delta, 2)$ is the unique irreducible generic subrepresentation of $\pi$ (see Section 3). We keep the notation of Section 5 and let $Q=P_{(n, n)}$, $L=M_{(n, n)}$, and $U=N_{(n, n)}$. Let $P=M N$ be a maximal $\Delta_{0}$-standard $\theta$ split parabolic subgroup of $G$, with unipotent radical $N$ and $\theta$-stable Levi factor $M=P \cap \theta(P)$. The Jacquet restriction functor (along $P$ ) is exact; therefore, we have an exact sequence of $M$-modules

$$
0 \rightarrow \mathcal{Z}(\delta, 2)_{N} \rightarrow \pi_{N} \rightarrow \mathcal{U}(\delta, 2)_{N} \rightarrow 0 .
$$

Our goal is to understand the irreducible subquotients, and the exponents, of $\mathcal{U}(\delta, 2)_{N}$ by applying the Geometric Lemma [BZ77, Lemma 2.12] to $\pi_{N}$. If $\chi \in$ $\operatorname{Exp}_{A_{M}}\left(\mathcal{U}(\delta, 2)_{N}\right)$, then $\chi$ is the central quasi-character of an irreducible subquotient of $\mathcal{U}(\delta, 2)_{N}$ and thus of $\pi_{N}$, that is, $\chi$ appears in $E_{x p_{A_{M}}}\left(\pi_{N}\right)$. Recall that we can realize $Q=w_{+}^{-1} P_{\Omega} w_{+}$, where $\Omega=\Delta_{0} \backslash\left\{w_{+}\left(\epsilon_{n}-\epsilon_{n+1}\right)\right\}$, and $P=P_{\Theta}$, for some $1 \leq k \leq n-1$, where $\Theta=\Theta_{k}$ is described in (5.1). In particular, $\Omega$ and $\Theta$ are subsets of the $\theta$-base $\Delta_{0}$. Let

$$
\left[W_{\Theta} \backslash W_{0} / W_{\Omega}\right]=\left\{w \in W_{0}: w \Omega \subset \Phi_{0}^{+}, w^{-1} \Theta \subset \Phi_{0}^{-1}\right\},
$$


where $\Phi_{0}^{+}$is the set of $\Delta_{0}$-positive roots. By [Cas95, Propositions 1.3 .1 and 1.3.3], the set $\left[W_{\Theta} \backslash W_{0} / W_{\Omega}\right] \cdot w_{+}$is a system of representatives for $P \backslash G / Q$. By the Geometric Lemma BZ77, Lemma 2.12], there exists a filtration of the space of $\pi_{N}$ such that the associated graded object $\operatorname{gr}\left(\pi_{N}\right)$ is isomorphic to

$$
\bigoplus_{y \in\left[W_{\Theta} \backslash W_{0} / W_{\Omega}\right] \cdot w_{+}} \iota_{M \cap^{y} Q}^{M}\left({ }^{y}\left(\nu^{1 / 2} \delta \otimes \nu^{-1 / 2} \delta\right)_{N \cap^{y} L}\right) .
$$

Write $\mathcal{F}_{N}^{y}(\delta, 2)$ to denote the representation $\iota_{M \cap^{y} Q}^{M}\left({ }^{y}\left(\nu^{1 / 2} \delta \otimes \nu^{-1 / 2} \delta\right)_{N \cap^{y} L}\right)$. Thus

$$
\operatorname{gr}\left(\pi_{N}\right) \cong \bigoplus_{w \in\left[W_{\Theta} \backslash W_{0} / W_{\Omega}\right]} \mathcal{F}_{N}^{w w_{+}}(\delta, 2)
$$

The exponents of $\pi$ along $P$ are the central characters of the irreducible subquotients of $\pi_{N}$; moreover, the exponents of $\mathcal{U}(\delta, 2)$ along $P$ are a subset of the exponents of $\pi$ along $P$. Recall that, by Lemma 5.1 the $(\theta, F)$-split component $S_{M}$ of $M$ is equal to its $F$-split component $A_{M}$; precisely,

$$
\begin{aligned}
A_{M} & =w_{+}\left\{\operatorname{diag}(\underbrace{a, \ldots, a}_{2 k}, \underbrace{b, \ldots, b}_{2 n-2 k}): a, b \in F^{\times}\right\} w_{+}^{-1} \\
& =\left\{\operatorname{diag}(\underbrace{a, \ldots, a}_{k}, \underbrace{b, \ldots, b}_{2 n-2 k}, \underbrace{a, \ldots, a}_{k}): a, b \in F^{\times}\right\} .
\end{aligned}
$$

By Cas95, Proposition 1.3.3], with our choice $\left[W_{\Theta} \backslash W_{0} / W_{\Omega}\right] \cdot w_{+}$of representatives for $P \backslash G / Q$, if $y=w w_{+}$where $w \in\left[W_{\Theta} \backslash W_{0} / W_{\Omega}\right]$, then $M \cap{ }^{y} Q$ is a parabolic subgroup of $M$ with Levi factor $M \cap{ }^{y} L$ and unipotent radical $M \cap{ }^{y} U$. Similarly, $P \cap{ }^{y} L$ is a parabolic subgroup of $L$ with Levi subgroup $M \cap{ }^{y} L$ and unipotent radical $N \cap{ }^{y} L$. Explicitly, since $P=P_{\Theta}$ and $Q=w_{+}^{-1} P_{\Omega} w_{+}$, we see that

$$
\begin{gathered}
M \cap{ }^{y} L=M_{\Theta} \cap w M_{\Omega} w^{-1}=M_{\Theta} \cap M_{w \Omega}=M_{\Theta \cap w \Omega}, \\
N \cap{ }^{y} L=N_{\Theta} \cap w M_{\Omega} w^{-1}=N_{\Theta} \cap M_{w \Omega},
\end{gathered}
$$

and

$$
M \cap{ }^{y} U=M_{\Theta} \cap w N_{\Omega} w^{-1}=M_{\Theta} \cap N_{w \Omega} .
$$

Let $w \in\left[W_{\Theta} \backslash W_{0} / W_{\Omega}\right]$. To achieve our goal, there are two cases that we need to consider.

Case 1: $P_{\Theta} \cap{ }^{w} M_{\Omega}={ }^{w} M_{\Omega}$.

Case 2: $P_{\Theta} \cap{ }^{w} M_{\Omega} \subsetneq{ }^{w} M_{\Omega}$ is a proper parabolic subgroup of ${ }^{w} M_{\Omega}$.

In Case 1, we show that the associated irreducible subquotients of $\pi_{N}$ are not $M^{\theta}$ distinguished. In Case 2, we show that the corresponding exponents of $\pi_{N}$ satisfy condition (2.2). The exact sequence (6.1) allows us to conclude that the same holds for $\mathcal{U}(\delta, 2)_{N}$.

6.1. Case 1: no distinction. Assume that $w \in\left[W_{\Theta} \backslash W_{0} / W_{\Omega}\right]$ is such that $P_{\Theta} \cap$ ${ }^{w} M_{\Omega}={ }^{w} M_{\Omega}$. Then $N_{\Theta} \cap{ }^{w} M_{\Omega}=\{e\}$ and $M_{\Theta} \cap{ }^{w} M_{\Omega}={ }^{w} M_{\Omega}=M_{w \Omega}$. In particular, ${ }^{w} M_{\Omega} \subset M_{\Theta}$, and since ${ }^{w} M_{\Omega}$ is maximal it follows that ${ }^{w} M_{\Omega}=M_{\Theta} \cong$ $\mathbf{G L}_{n}(F) \times \mathbf{G L}_{n}(F)$. That is, $M_{\Theta}$ and $M_{\Omega}$ are associate standard Levi subgroups isomorphic to $\mathbf{G L}_{n}(F) \times \mathbf{G L}_{n}(F)$. It follows that $n$ must be even, $k=n / 2$, and $\Theta=\Theta_{n / 2}=w_{+}\left(\Delta \backslash\left\{\epsilon_{n}-\epsilon_{n+1}\right\}\right)=\Omega$. That is, $M_{\Theta}=M_{\Omega}$ and $w$ lies in 
$\left[W_{\Omega} \backslash W_{0} / W_{\Omega}\right] \cap W(\Omega, \Omega)$, where $W(\Theta, \Omega)=\left\{w \in W_{0}: w \Omega=\Theta\right\}$. Set $y=w w_{+}$. Then $M_{\Omega} \cap{ }^{y} Q=M_{w \Omega}=M_{\Omega}$ and $P_{\Omega} \cap{ }^{y} L=M_{w \Omega}=M_{\Omega}$. In this setting,

$$
\mathcal{F}_{\Omega}^{y}(\delta, 2)=\iota_{M_{\Omega}}^{M_{\Omega}}\left({ }^{y}\left(\nu^{1 / 2} \delta \otimes \nu^{-1 / 2} \delta\right)_{\{e\}}\right)={ }^{y}\left(\nu^{1 / 2} \delta \otimes \nu^{-1 / 2} \delta\right),
$$

since $N_{\Omega} \cap{ }^{w} M_{\Omega}=N_{\Omega} \cap M_{\Omega}=\{e\}$.

Proposition 6.2. Let $w \in\left[W_{\Omega} \backslash W_{0} / W_{\Omega}\right] \cap W(\Omega, \Omega)$ and set $y=w w_{+}$. Let $\tau$ be an irreducible admissible generic representation of $\mathbf{G L}_{n}(F)$. The representation ${ }^{y}\left(\nu^{1 / 2} \tau \otimes \nu^{-1 / 2} \tau\right)$ of $M_{\Omega}$ is not $M_{\Omega}^{\theta}$-distinguished, that is, $\operatorname{Hom}_{M_{\Omega}^{\theta}}\left(y\left(\nu^{1 / 2} \tau \otimes \nu^{-1 / 2} \tau\right), 1\right)=0$.

Proof. First, recall that $n$ is even, and observe that $M_{\Omega}^{\theta} \cong \mathbf{S p}_{n}(F) \times \mathbf{S p}_{n}(F)$. Indeed, $M_{\Omega}=w_{+} M_{(n, n)} w_{+}^{-1}$ and $m=w_{+} \underline{m} w_{+}^{-1} \in M_{\Omega}$ is $\theta$-fixed if and only if $\underline{m} \in M_{(n, n)}$ is fixed by $w_{+} \cdot \theta=\theta_{x_{2 n}}$. Recall (see Section 5) that

$$
x_{2 n}=\varepsilon_{2 n} \cdot w_{+}=\left(\begin{array}{ccccc}
0 & 1 & & & \\
-1 & 0 & & & \\
& & \ddots & & \\
& & & 0 & 1 \\
& & & -1 & 0
\end{array}\right) \in M_{(n, n)}
$$

and $\theta_{x_{2 n}}(g)=x_{2 n}^{-1 t} g^{-1} x_{2 n}$. One may readily verify that the image of $\underline{m}=$ $\operatorname{diag}\left(m_{1}, m_{2}\right) \in M_{(n, n)}$ under $\theta_{x_{2 n}}$ is given by

$$
\theta_{x_{2 n}}(\underline{m})=\operatorname{diag}\left(x_{n}^{-1 t} m_{1}^{-1} x_{n}, x_{n}^{-1 t} m_{2}^{-1} x_{n}\right)=\operatorname{diag}\left(\theta_{x_{n}}\left(m_{1}\right), \theta_{x_{n}}\left(m_{2}\right)\right) .
$$

It follows that $\underline{m}$ is $\theta_{x_{2 n}}$-fixed if and only if $m_{i}=\theta_{x_{n}}\left(m_{i}\right)$, for each $i=1,2$. Moreover,

$$
\begin{aligned}
M_{\Omega}^{\theta} & =w_{+}\left(M_{(n, n)}^{\theta_{x_{2 n}}}\right) w_{+}^{-1} \\
& =w_{+}\left(\mathbf{G L}_{n}(F)^{\theta_{x_{n}}} \times \mathbf{G L}_{n}(F)^{\theta_{x_{n}}}\right) w_{+}^{-1} \\
& \cong\left(\mathbf{S p}_{n}(F) \times \mathbf{S p}_{n}(F)\right),
\end{aligned}
$$

since $x_{n} \in \mathbf{G L}_{n}(F)$ is nonsingular and skew symmetric, and $\mathbf{G L}_{n}^{\theta_{x_{n}}} \cong \mathbf{S p}_{n}$.

Next, we note that $\left[W_{\Omega} \backslash W_{0} / W_{\Omega}\right] \cap W(\Omega, \Omega)$ consists of two elements: the identity $e$ and $w_{+} w_{(n, n)} w_{+}^{-1}$, where

$$
w_{(n, n)}=\left(\begin{array}{cc}
0 & I_{n} \\
I_{n} & 0
\end{array}\right)
$$

First, realize $\left[W_{\Omega} \backslash W_{0} / W_{\Omega}\right]=w_{+}\left[W_{\Omega^{\mathrm{el}} \backslash} \backslash W_{0} / W_{\Omega^{\mathrm{ell}}}\right] w_{+}^{-1}$, and $W(\Omega, \Omega)=$ $w_{+} W\left(\Omega^{\text {ell }}, \Omega^{\text {ell }}\right) w_{+}^{-1}$, where $\Omega^{\text {ell }}=\Delta \backslash\left\{\epsilon_{n}-\epsilon_{n+1}\right\}=w_{+}^{-1} \Omega$. If $w \in W_{0}$, then we identify $w$ with a permutation of $\{1, \ldots, 2 n\}$ and note that $w\left(\epsilon_{i}\right)=\epsilon_{w(i)}$. The set of $\Delta$-positive roots in $\Phi_{0}$ is $\Phi_{\Delta}^{+}=\left\{\epsilon_{i}-\epsilon_{j}: 1 \leq i<j \leq 2 n\right\}$. Thus, by definition, $w \in W_{0}$ lies in the set $\left[W_{\Omega^{\text {ell }}} \backslash W_{0} / W_{\Omega^{\text {ell }}}\right]$ if and only if $w(i)<w(i+1)$ and $w^{-1}(i)<w^{-1}(i+1)$, for all $1 \leq i \leq n-1$ and $n+1 \leq i \leq 2 n-1$ (with $i \neq n$ since $\left.\epsilon_{n}-\epsilon_{n+1} \notin \Omega^{\text {ell }}\right)$. It is not difficult to verify that $\left[W_{\Omega^{\text {ell }}} \backslash W_{0} / W_{\Omega^{\text {ell }}}\right]$ consists of the $n+1$ permutation matrices of the form

$$
\left(\begin{array}{cccc}
I_{j} & 0 & 0 & 0 \\
0 & 0 & I_{n-j} & 0 \\
0 & I_{n-j} & 0 & 0 \\
0 & 0 & 0 & I_{j}
\end{array}\right)
$$


where $0 \leq j \leq n$. Notice that $j=0$ corresponds to $w_{(n, n)}$ and $j=n$ corresponds to the identity matrix $e=I_{2 n}$. On the other hand, the elements of the set $W\left(\Omega^{\text {ell }}, \Omega^{\text {ell }}\right)$ satisfy $w \Omega^{\text {ell }}=\Omega^{\text {ell }}$ and thus normalize the block-diagonal Levi subgroup $M_{(n, n)}=$ $M_{\Omega^{\text {ell }}}$. One may quickly check that, of the elements of the form in (6.3), only the identity $e$ and $w_{(n, n)}$ normalize $M_{(n, n)}$. It follows that $\left[W_{\Omega^{\text {ell }}} \backslash W_{0} / W_{\Omega^{\text {ell }}}\right] \cap$ $W\left(\Omega^{\mathrm{ell}}, \Omega^{\mathrm{ell}}\right)$ consists of precisely $e$ and $w_{(n, n)}$, proving the claim.

We now turn to studying the $M_{\Omega^{-}}^{\theta}$ distinction of $\mathcal{F}_{\Omega}^{y}(\tau, 2)={ }^{y}\left(\nu^{1 / 2} \tau \otimes \nu^{-1 / 2} \tau\right)$, where $y=w w_{+}$. There are two sub-cases to consider, either $w=e$ or $w=$ $w_{+} w_{(n, n)} w_{+}^{-1}$. If $w=e$, then $y=w_{+} \in\left[W_{\Omega} \backslash W_{0} / W_{\Omega}\right] \cap W(\Omega, \Omega)$. As above, $\mathscr{F}_{\Omega}^{y}(\tau, 2)={ }^{w_{+}}\left(\nu^{1 / 2} \tau \otimes \nu^{-1 / 2} \tau\right)$. If $w=w_{(n, n)}$, then $y=w w_{+}=w_{+} w_{(n, n)} w_{+}^{-1} w_{+}=$ $w_{+} w_{(n, n)}$. It follows that $\mathscr{F}_{\Omega}^{y}(\tau, 2)=w_{+} w_{(n, n)}\left(\nu^{1 / 2} \tau \otimes \nu^{-1 / 2} \tau\right)$. Conjugation by $w_{(n, n)}$ interchanges the two GL-blocks of $M_{\Omega}=M_{(n, n)}$; therefore, twisting a representation $\pi_{1} \otimes \pi_{2}$ of $M_{\Omega}$ by $w_{(n, n)}$ interchanges the two representations, that is, $w_{(n, n)}\left(\pi_{1} \otimes \pi_{2}\right) \cong \pi_{2} \otimes \pi_{1}$. Therefore, ${ }^{w_{+} w_{(n, n)}}\left(\nu^{1 / 2} \tau \otimes \nu^{-1 / 2} \tau\right)={ }^{w_{+}}\left(\nu^{-1 / 2} \tau \otimes \nu^{1 / 2} \tau\right)$. We have seen above that $M_{\Omega}^{\theta} \cong\left(\mathbf{S p}_{n}(F) \times \mathbf{S p}_{n}(F)\right)$. In both cases $(w=e, w=$ $\left.w_{(n, n)}\right)$, it follows that $\mathcal{F}_{\Omega}^{y}(\tau, 2)$ is $M_{\Omega}^{\theta}$-distinguished if and only if $\nu^{1 / 2} \tau$ and $\nu^{-1 / 2} \tau$ are $\mathbf{S p}_{n}(F)$-distinguished. By assumption, $\tau$ is an irreducible generic representation; therefore, by [HR90, Theorem 3.2.2], $\operatorname{Hom}_{\mathbf{S p}_{n}(F)}(\tau, 1)=\{0\}$. It follows, since $\nu$ is trivial on (maximal) unipotent subgroups of $\mathbf{G} \mathbf{L}_{n}(F)$, that $\nu^{s} \tau$ is generic and $\operatorname{Hom}_{\mathbf{S p}_{n}(F)}\left(\nu^{s} \tau, 1\right)=\{0\}$, for every $s \in \mathbb{C}$. Moreover, if $w$ is equal to either $e$ or $w_{(n, n)}$, then $\operatorname{Hom}_{M_{\Omega}^{\theta}}\left(\mathcal{F}_{\Omega}^{y}(\tau, 2), 1\right)=0$, as claimed.

6.2. Case 2: 'good' exponents. Assume that $w \in\left[W_{\Theta} \backslash W_{0} / W_{\Omega}\right]$ is such that $P_{\Theta} \cap{ }^{w} M_{\Omega}$ is a proper parabolic subgroup of ${ }^{w} M_{\Omega}$. First, we show that $M_{\Theta} \cap{ }^{w} P_{\Omega}$ is also a proper parabolic subgroup of $M_{\Theta}$. We argue by contradiction, and suppose that $M_{\Theta} \cap{ }^{w} P_{\Omega}=M_{\Theta}$. By Cas95, Proposition 1.3.3], $M_{\Theta} \cap{ }^{w} N_{\Omega}=\{e\}$ and $M_{\Theta} \cap{ }^{w} M_{\Omega}=M_{\Theta}$. In particular, $M_{\Theta} \subset{ }^{w} M_{\Omega}=M_{w \Omega}$. However, both $M_{\Omega}$ and $M_{\Theta}$ are maximal Levi subgroups of $G$, and it follows that $M_{\Theta}={ }^{w} M_{\Omega}$. This, in turn, implies that $P_{\Theta} \cap{ }^{w} M_{\Omega}=M_{\Theta}={ }^{w} M_{\Omega}$, which contradicts our assumption that $P_{\Theta} \cap{ }^{w} M_{\Omega}$ is a proper parabolic subgroup of ${ }^{w} M_{\Omega}$. We conclude that $M_{\Theta} \cap{ }^{w} P_{\Omega}$ is a proper parabolic subgroup of $M_{\Theta}$.

It follows from this last observation that if $y=w w_{+}$, then the representation $\mathcal{F}_{N}^{y}(\delta, 2)=\iota_{M \cap y}^{M}\left({ }^{y}\left(\nu^{1 / 2} \delta \otimes \nu^{-1 / 2} \delta\right)_{N \cap^{y} L}\right)$ is induced from ${ }^{y}\left(\nu^{1 / 2} \delta \otimes \nu^{-1 / 2} \delta\right)_{N \cap^{y} L}$ along the proper parabolic $M \cap{ }^{y} Q=M_{\Theta} \cap{ }^{w} P_{\Omega}$ of $M=M_{\Theta}$; moreover, the Jacquet module ${ }^{y}\left(\nu^{1 / 2} \delta \otimes \nu^{-1 / 2} \delta\right)_{N \cap^{y} L}$ is taken along the proper parabolic $P \cap^{y} L=$ $P_{\Theta} \cap{ }^{w} M_{\Omega}$ of ${ }^{y} L={ }^{w} M_{\Omega}$. That is, both the Jacquet restriction and parabolic induction steps appearing in $\mathcal{F}_{N}^{y}(\delta, 2)$ are along proper parabolic subgroups. To be completely explicit, we note that

$$
\mathcal{F}_{N}^{w w_{+}}(\delta, 2)=\iota_{M_{\Theta} \cap w}^{M_{\Theta}} P_{\Omega}\left(w w_{+}\left(\nu^{1 / 2} \delta \otimes \nu^{-1 / 2} \delta\right)_{N_{\Theta} \cap w} M_{\Omega}\right) .
$$

In this subsection, we will use the shorthand notation $[\tau]=\nu^{1 / 2} \tau \otimes \nu^{-1 / 2} \tau$, where $\tau$ is an irreducible admissible representation of $\mathbf{G} \mathbf{L}_{n}(F)$. Our goal is to compute the exponents of $\pi=\nu^{1 / 2} \delta \times \nu^{-1 / 2} \delta$ along $P=P_{\Theta}$; therefore, we need to understand the central characters of the irreducible subquotients of the $\mathscr{F}^{w w_{+}}(\delta, 2)$. By Smi18b, Lemma 4.16], the quasi-characters appearing in $\mathscr{E} x p_{A_{\Theta}}\left(\mathscr{F}^{w w_{+}}(\delta, 2)\right)$ are the restrictions to $A_{\Theta}$ of the quasi-characters appearing in $\delta x p_{A_{\Theta \cap w_{\Omega}}}\left(w w_{+}[\delta]_{N_{\Theta} \cap w} M_{\Omega}\right)$, where 
the $F$-split component of $M_{\Theta} \cap{ }^{w} M_{\Omega}=M_{\Theta \cap w \Omega}$ is $A_{\Theta \cap w \Omega}$. Thus, our problem reduces to understanding the exponents of $w w_{+}[\delta]$ along $P_{\Theta} \cap{ }^{w} M_{\Omega}$.

Since $L=M_{(n, n)} \cong \mathbf{G L}_{n}(F) \times \mathbf{G L}_{n}(F)$, we have that $P_{\Theta} \cap{ }^{w} M_{\Omega} \cong P_{1} \times P_{2}$, where $P_{1}$ and $P_{2}$ are parabolic subgroups of $\mathbf{G L}_{n}(F)$, at least one of which is proper. We can realize $w=w_{+} w^{\prime} w_{+}^{-1} \in\left[W_{\Theta} \backslash W_{0} / W_{\Omega}\right]$, where $w^{\prime} \in\left[W_{(2 k, 2 n-2 k)} \backslash W_{0} / W_{(n, n)}\right]$. Then, with $w=w_{+} w^{\prime} w_{+}^{-1}$,

$$
\begin{aligned}
& \left({ }^{w w+}[\delta]\right)_{N_{\Theta} \cap{ }^{w} M_{\Omega}}=\left(w_{+} w^{\prime}[\delta]\right)_{w_{+} N_{(2 k, 2 n-2 k)} w_{+}^{-1} \cap w_{+} w^{\prime} M_{(n, n)} w^{-1} w_{+}^{-1}} \\
& =\left(w_{+} w^{\prime}[\delta]\right)_{w_{+}\left(N_{(2 k, 2 n-2 k)} \cap w^{\prime} M_{(n, n)} w^{\prime-1}\right) w_{+}^{-1}} \\
& =w_{+}\left(w^{\prime}[\delta]_{N_{(2 k, 2 n-2 k)} \cap w^{\prime} M_{(n, n)} w^{\prime-1}}\right) \\
& =w_{+} w^{\prime}\left([\delta]_{w^{\prime-1} N_{(2 k, 2 n-2 k)} w^{\prime} \cap M_{(n, n)}}\right) \\
& =w_{+} w^{\prime}\left([\delta]_{N_{1} \times N_{2}}\right) \text {, }
\end{aligned}
$$

where we identify $P_{\Theta} \cap{ }^{w} M_{\Omega} \cong P_{1} \times P_{2}$ with a parabolic subgroup of $M_{(n, n)} \cong$ $\mathbf{G L}_{n}(F) \times \mathbf{G L}_{n}(F)$ via

$$
\begin{aligned}
& P_{1} \times P_{2}=w^{\prime-1} N_{(2 k, 2 n-2 k)} w^{\prime} \cap M_{(n, n)} \\
& =w^{\prime-1}\left(N_{(2 k, 2 n-2 k)} \cap w^{\prime} M_{(n, n)} w^{\prime-1}\right) w^{\prime} \\
& =w^{\prime-1} w_{+}^{-1}\left(w_{+} N_{(2 k, 2 n-2 k)} w_{+}^{-1} \cap w_{+} w^{\prime} M_{(n, n)} w^{\prime-1} w_{+}^{-1}\right) w_{+} w^{\prime} \\
& =w^{\prime-1} w_{+}^{-1}\left(w_{+} N_{(2 k, 2 n-2 k)} w_{+}^{-1} \cap w_{+} w^{\prime} w_{+}^{-1} w_{+} M_{(n, n)} w_{+}^{-1} w_{+} w^{\prime-1} w_{+}^{-1}\right) w_{+} w^{\prime} \\
& =w^{\prime-1} w_{+}^{-1}\left(w_{+} N_{(2 k, 2 n-2 k)} w_{+}^{-1} \cap w w_{+} M_{(n, n)} w_{+}^{-1} w^{-1}\right) w_{+} w^{\prime} \\
& =w^{-1} w_{+}^{-1}\left(N_{\Theta} \cap w M_{\Omega} w^{-1}\right) w_{+} w^{\prime} \\
& =w_{+}^{-1} w^{-1}\left(N_{\Theta} \cap w M_{\Omega} w^{-1}\right) w_{+} w,
\end{aligned}
$$

using that $w w_{+}=w_{+} w^{\prime} w_{+}^{-1} w_{+}=w_{+} w^{\prime}$. It follows that

$$
\begin{aligned}
\left({ }^{w w+}[\delta]\right)_{N_{\Theta} \cap{ }^{w} M_{\Omega}} & ={ }^{w_{+} w^{\prime}}\left([\delta]_{N_{1} \times N_{2}}\right) \\
& ={ }^{w_{+} w^{\prime}}\left(\left(\nu^{1 / 2} \delta \otimes \nu^{-1 / 2} \delta\right)_{N_{1} \times N_{2}}\right) \\
& ={ }^{w_{+} w^{\prime}}\left(\nu^{1 / 2} \delta_{N_{1}} \otimes \nu^{-1 / 2} \delta_{N_{2}}\right) \\
& =w w_{+}\left(\nu^{1 / 2} \delta_{N_{1}} \otimes \nu^{-1 / 2} \delta_{N_{2}}\right),
\end{aligned}
$$

where in the final equality we have again used that $w w_{+}=w_{+} w^{\prime}$. In the above calculation of $\left({ }^{w w+}[\delta]\right)_{N_{\Theta} \cap^{w} M_{\Omega}}$, we also implicitly used the following basic fact.

Lemma 6.3. Let $(\pi, V)$ be a smooth representation of $G=\mathbf{G L}_{m}(F)$. Let $P=M N$ be a (proper) parabolic subgroup of $G$ with Levi factor $M$ and unipotent radical $N$. Let $s \in \mathbb{C}$. Then the Jacquet module $\left(\nu^{s} \otimes \pi\right)_{N}$ is equivalent to the twisted Jacquet $\left.\operatorname{module} \nu^{s}\right|_{M} \otimes \pi_{N}$.

Proof. The lemma follows immediately from the fact that $\nu$ is trivial on the unipotent group $N$. Indeed, the space of both representations $\pi$ and $\nu^{s} \otimes \pi=\nu^{s} \pi$ is $V$. The space of the Jacquet module of $\pi$, respectively $\nu^{s} \pi$, is the quotient of $V$ by the subspace $V(N)=\operatorname{span}\{v-\pi(n) v: v \in V, n \in N\}$, respectively $\operatorname{span}\left\{v-\nu^{s}(n) \pi(n) v: v \in V, n \in N\right\}$. Since $\nu^{s}(n)=1$ for every $n \in N$, we see that 
the space of both $\pi_{N}$ and $\left(\nu^{s} \pi\right)_{N}$ is $V_{N}=V / V(N)$. Finally, observe that for any $m \in M$ and $v+V(N) \in V_{N}$ we have

$$
\begin{aligned}
\left(\nu^{s} \pi\right)_{N}(m)(v+V(N)) & =\delta_{P}^{-1 / 2}(m) \nu^{s}(m) \pi(m) v+V(N) \\
& =\nu^{s}(m)\left(\delta_{P}^{-1 / 2}(m) \pi(m) v+V(N)\right) \\
& =\nu^{s}(m) \pi_{N}(m)(v+V(N)) ;
\end{aligned}
$$

therefore $\left(\nu^{s} \pi\right)_{N}=\left.\nu^{s}\right|_{M} \otimes \pi_{N}$, as claimed.

In order to understand the exponents of $(w w+[\delta])_{N_{\Theta} \cap w M_{\Omega}}$, we require the following proposition.

Proposition 6.4. Let $G$ and $G^{\prime}$ be two connected reductive groups over $F$. Let $(\pi, V)$, respectively $(\sigma, W)$, be a finitely generated admissible representation of $G$, respectively $G^{\prime}$. The set of exponents of the (external) tensor product $\pi \otimes \sigma$ consists of all pairwise products $\chi \otimes \chi^{\prime}$, where $\chi \in \mathcal{E}_{x} p_{Z_{G}}(\pi)$ and $\chi^{\prime} \in \mathcal{E}_{x} p_{Z_{G^{\prime}}}(\sigma)$ are exponents of $\pi$ and $\sigma$ respectively. That is,

$$
\begin{aligned}
\mathscr{E x p}_{Z_{G} \times Z_{G^{\prime}}}(\pi \otimes \sigma) & =\left\{\chi \otimes \chi^{\prime}: \chi \in \mathcal{E}_{x} p_{Z_{G}}(\pi), \chi^{\prime} \in \mathcal{E}_{x} p_{Z_{G^{\prime}}}(\sigma)\right\} \\
& \cong \mathcal{E}_{x} p_{Z_{G}}(\pi) \times \mathscr{E}_{x} p_{Z_{G^{\prime}}}(\sigma) .
\end{aligned}
$$

Proof. The exponents $\varepsilon x p_{Z_{G} \times Z_{G^{\prime}}}(\pi \otimes \sigma)$ of $\pi \otimes \sigma$ are precisely the central characters of the irreducible subquotients of $\pi \otimes \sigma$ (cf. [Cas95, Proposition 2.1.9], SSmi18b, Lemma 4.14]). To prove the proposition, it is sufficient to show that the irreducible subquotients of $\pi \otimes \sigma$ are of the form $V^{j} \otimes W^{k}$, where $V^{j}$, respectively $W^{k}$, is an irreducible subquotient of $(\pi, V)$, respectively $(\sigma, W)$. Indeed, if $V^{j}$ (resp. $\left.W^{k}\right)$ is irreducible, then it admits a central character $\chi_{j}\left(\right.$ resp. $\left.\chi_{k}\right)$; moreover, $V^{j} \otimes W^{k}$ has central character $\chi_{j} \otimes \chi_{k}: Z_{G} \times Z_{G^{\prime}} \rightarrow \mathbb{C}^{\times}$. We omit the proof of the elementary fact regarding the subquotients of the external tensor product $(\pi \otimes \sigma, V \otimes W)$.

Note. To clarify the following calculations we introduce some additional notation for certain subsets of $\Delta$. For and $1 \leq j \leq 2 n-1$, let $\Xi_{j}=\Delta \backslash\left\{\epsilon_{j}-\epsilon_{j+1}\right\}$. We will be particularly interested in $\Xi_{2 k}$ and $\Xi_{n}=\Omega^{\text {ell }}$ since $\Theta=w_{+} \Xi_{2 k}$ and $\Omega=w_{+} \Xi_{n}$.

Recall that the $(\theta, F)$-split component $S_{\Theta}$ of $M_{\Theta}$ is equal to the $F$-split component $A_{\Theta}$. In particular, the $(\theta, F)$-split component of $G$ is $S_{G}=A_{G}$. We now consider the exponents of $\left({ }^{w w+}[\delta]\right)_{N_{\Theta} \cap{ }^{w} M_{\Omega}}={ }^{w w_{+}}\left(\nu^{1 / 2} \delta_{N_{1}} \otimes \nu^{-1 / 2} \delta_{N_{2}}\right)$ restricted to $S_{\Theta}^{-} \backslash S_{\Theta}^{1} S_{G}=A_{\Theta}^{-} \backslash A_{\Theta}^{1} A_{G}$. Let $s \in S_{\Theta}=A_{\Theta}$. Since $A_{\Theta}=w_{+} A_{(2 k, 2 n-2 k)} w_{+}^{-1}$, we can write $s=w_{+} a w_{+}^{-1}$, where $a=\operatorname{diag}\left(a_{1} I_{2 k}, a_{2} I_{2 n-2 k}\right)$ lies in $A_{(2 k, 2 n-2 k)}^{-} \backslash$ $A_{(2 k, 2 n-2 k)}^{1} A_{G}$. In particular, $A_{(2 k, 2 n-2 k)}=A_{\Xi_{2 k}}$ and $a$ has the property that $\left|\epsilon_{2 k}-\epsilon_{2 k+1}(a)\right|=\left|a_{1} a_{2}^{-1}\right|<1$. By Proposition 6.4 and Lemma 6.3, the exponents of $(w w+[\delta])_{N_{\Theta} \cap w} M_{\Omega}=w w_{+}\left(\nu^{1 / 2} \delta_{N_{1}} \otimes \nu^{-1 / 2} \delta_{N_{2}}\right)$ are all of the form $w w_{+}\left(\nu^{1 / 2} \chi_{1} \otimes \nu^{-1 / 2} \chi_{2}\right)$, where $\chi_{1} \in \mathcal{E} x p_{A_{1}}\left(\delta_{N_{1}}\right)$, and $\chi_{2} \in \mathcal{E} x p_{A_{2}}\left(\delta_{N_{2}}\right)$. Here we write $A_{i}$ for the $F$-split component of $M_{i} \subset P_{i} \subset \mathbf{G L}_{n}(F), i=1,2$. In particular,

$$
\begin{aligned}
w w_{+}\left(\nu^{1 / 2} \chi_{1} \otimes \nu^{-1 / 2} \chi_{2}\right)(s) & ={ }^{w w_{+}}\left(\nu^{1 / 2} \chi_{1} \otimes \nu^{-1 / 2} \chi_{2}\right)\left(w_{+} a w_{+}^{-1}\right) \\
& =\left(\nu^{1 / 2} \chi_{1} \otimes \nu^{-1 / 2} \chi_{2}\right)\left(w_{+}^{-1} w^{-1} w_{+} a w_{+}^{-1} w w_{+}\right) \\
& =\left(\nu^{1 / 2} \chi_{1} \otimes \nu^{-1 / 2} \chi_{2}\right)\left(w^{\prime-1} a w^{\prime}\right),
\end{aligned}
$$


where $w^{\prime}=w_{+}^{-1} w w_{+} \in\left[W_{(2 k, 2 n-2 k)} \backslash W_{0} / W_{(n, n)}\right]$ and

$$
\begin{aligned}
w^{\prime-1} a w^{\prime} & \in w^{\prime-1} A_{(2 k, 2 n-2 k)}^{-} w^{\prime} \backslash w^{\prime-1} A_{(2 k, 2 n-2 k)}^{1} w^{\prime} A_{G} \\
& \subset A_{w^{\prime-1} M_{(2 k, 2 n-2 k)} w^{\prime} \cap M_{(n, n)}} \backslash A_{w^{\prime-1} M_{(2 k, 2 n-2 k)} w^{\prime} \cap M_{(n, n)}}^{1} A_{(n, n)} \\
& =A_{\left(w^{\prime-1} \Xi_{2 k}\right) \cap \Xi_{n}}^{-} \backslash A_{\left(w^{\prime-1} \Xi_{2 k}\right) \cap \Xi_{n}}^{1} A_{(n, n)} \\
& =A_{M_{1} \times M_{2}}^{-} \backslash A_{M_{1} \times M_{2}}^{1} A_{(n, n)} \\
& =A_{1}^{-} \times A_{2}^{-} \backslash\left(A_{1}^{1} \times A_{2}^{1}\right) A_{(n, n)},
\end{aligned}
$$

where the containment in the second line follows as in the proof of Smi18b, Lemma 8.4]. By assumption, $\delta$ is a discrete series representation of $\mathbf{G L}_{n}(F)$; therefore, the exponents $\chi_{1}$ and $\chi_{2}$ of $\delta$ satisfy Casselman's Criterion (Cas95, Theorem 6.5.1]) and

$$
\left|\chi_{1} \otimes \chi_{2}\left(w^{\prime-1} a w^{\prime}\right)\right|<1 .
$$

To ensure that the exponents $w w_{+}\left(\nu^{1 / 2} \chi_{1} \otimes \nu^{-1 / 2} \chi_{2}\right)$ of $(w w+[\delta])_{N_{\Theta} \cap{ }^{w} M_{\Omega}}=$ $w w_{+}\left(\nu^{1 / 2} \delta_{N_{1}} \otimes \nu^{-1 / 2} \delta_{N_{2}}\right)$ satisfy the Relative Casselman's Criterion (K10, Theorem 4.7]), we need to ensure that

$$
\left|\nu^{1 / 2} \otimes \nu^{-1 / 2}\left(w^{\prime-1} a w^{\prime}\right)\right| \leq 1 .
$$

We can realize the restriction of the unramified character $\nu^{1 / 2} \otimes \nu^{-1 / 2}$ to the maximal (diagonal) $F$-split torus $A_{0}$ as the composition of $|\cdot|_{F}^{1 / 2}$ with the sum over all roots in $\Delta$ with positive integral coefficients, that is,

$$
\left.\left(\nu^{1 / 2} \otimes \nu^{-1 / 2}\right)\right|_{A_{0}}=|\cdot|_{F}^{1 / 2} \circ\left(\sum_{\alpha \in \Delta} c_{\alpha} \cdot \alpha\right),
$$

where $c_{\epsilon_{i}-\epsilon_{i+1}}=i$, for $1 \leq i \leq n$, and $c_{\epsilon_{n+j}-\epsilon_{n+j+1}}=n-j$, for $1 \leq j \leq n-1$. To compute $\left(\nu^{1 / 2} \otimes \nu^{-1 / 2}\right)\left(w^{\prime-1} a w^{\prime}\right)$ it is helpful to partition $\Delta$ as the disjoint union of $\left(w^{\prime-1} \Xi_{2 k}\right) \cap \Xi_{n}$ and $\Delta \backslash\left(\left(w^{\prime-1} \Xi_{2 k}\right) \cap \Xi_{n}\right)$. Indeed, since $A_{w^{\prime-1} \Xi_{2 k}} \subset$ $A_{\left(w^{\prime-1} \Xi_{2 k}\right) \cap \Xi_{n}}$, it follows that $\alpha\left(w^{\prime-1} a w^{\prime}\right)=1$, for all $\alpha \in\left(w^{\prime-1} \Xi_{2 k}\right) \cap \Xi_{n}$. On the other hand, since $w^{\prime-1} a w^{\prime} \in A_{\left(w^{\prime-1} \Xi_{2 k}\right) \cap \Xi_{n}}^{-}$we have that $\left|\beta\left(w^{\prime-1} a w^{\prime}\right)\right|_{F} \leq 1$, for all $\beta \in \Delta \backslash\left(\left(w^{\prime-1} \Xi_{2 k}\right) \cap \Xi_{n}\right)$. From (6.6) , it follows that

$$
\begin{aligned}
& \left(\nu^{1 / 2} \otimes \nu^{-1 / 2}\right)\left(w^{\prime-1} a w^{\prime}\right)=\prod_{\alpha \in \Delta}\left|\alpha\left(w^{\prime-1} a w^{\prime}\right)\right|_{F}^{c_{\alpha} / 2} \\
& =\left(\prod_{\alpha \in\left(w^{\prime}-1 \Xi_{2 k}\right) \cap \Xi_{n}}\left|\alpha\left(w^{\prime-1} a w^{\prime}\right)\right|_{F}^{c_{\alpha} / 2}\right)\left(\prod_{\beta \in \Delta \backslash\left(\left(w^{\prime-1} \Xi_{2 k}\right) \cap \Xi_{n}\right)}\left|\beta\left(w^{\prime-1} a w^{\prime}\right)\right|_{F}^{c_{\beta} / 2}\right) \\
& =\prod_{\beta \in \Delta \backslash\left(\left(w^{\prime-1} \Xi_{2 k}\right) \cap \Xi_{n}\right)}\left|\beta\left(w^{\prime-1} a w^{\prime}\right)\right|_{F}^{c_{\beta} / 2} \\
& \leq 1,
\end{aligned}
$$

which establishes the truth of (6.5). Moreover, we now have that

$$
\begin{aligned}
\left|w w_{+}\left(\nu^{1 / 2} \chi_{1} \otimes \nu^{-1 / 2} \chi_{2}\right)(s)\right| & =\left(\nu^{1 / 2} \chi_{1} \otimes \nu^{-1 / 2} \chi_{2}\right)\left(w^{\prime-1} a w^{\prime}\right) \\
& =\left|\chi_{1} \otimes \chi_{2}\left(w^{\prime-1} a w^{\prime}\right)\right|\left|\nu^{1 / 2} \otimes \nu^{-1 / 2}\left(w^{\prime-1} a w^{\prime}\right)\right| \\
& <1,
\end{aligned}
$$


for all $\chi_{1} \in \mathcal{E} x p_{A_{1}}\left(\delta_{N_{1}}\right), \chi_{2} \in \mathcal{E} x p_{A_{2}}\left(\delta_{N_{2}}\right)$, and $s=w_{+} a w_{+}^{-1} \in S_{\Theta} \backslash S_{\Theta}^{1} S_{G}$, where $w^{\prime}=w_{+}^{-1} w w_{+}$as above. Finally, we have established the desired result:

Proposition 6.5. Let $\Theta=\Theta_{k}, 1 \leq k \leq n-1$, be a maximal $\theta$-split subset of $\Delta_{0}$. Let $w \in\left[W_{\Theta} \backslash W_{0} / W_{\Omega}\right]$ be such that $P_{\Theta} \cap{ }^{w} M_{\Omega}$ is a proper parabolic subgroup of ${ }^{w} M_{\Omega}$. Let $\delta$ be an irreducible admissible square integrable representation of $\mathbf{G L}_{n}(F)$. The exponents of $\pi_{N}$, and $\mathcal{U}(\delta, 2)_{N}$, corresponding to the irreducible subquotients of $\mathcal{F}_{N}^{w w_{+}}(\delta, 2)=\left({ }^{w w+}[\delta]\right)_{N_{\Theta} \cap{ }^{w} M_{\Omega}}$ satisfy condition (2.2) of Theorem 2.7.

\section{ACKNOWLEDGMENTS}

The author would like to thank Omer Offen and Yiannis Sakellaridis for many helpful discussions. The author also thanks the anonymous referee for several helpful suggestions.

\section{REFERENCES}

[Art13] James Arthur, The endoscopic classification of representations: Orthogonal and symplectic groups, American Mathematical Society Colloquium Publications, vol. 61, American Mathematical Society, Providence, RI, 2013. MR.3135650

[BD08] Philippe Blanc and Patrick Delorme, Vecteurs distributions H-invariants de représentations induites, pour un espace symétrique réductif p-adique $G / H$ (French, with English and French summaries), Ann. Inst. Fourier (Grenoble) 58 (2008), no. 1, 213-261. MR 2401221

[BZ77] I. N. Bernstein and A. V. Zelevinsky, Induced representations of reductive $\mathfrak{p}$-adic groups. I, Ann. Sci. École Norm. Sup. (4) 10 (1977), no. 4, 441-472. MR579172

[Cas95] William Casselman, Introduction to the theory of admissible representations of $p$-adic reductive groups, Unpublished manuscript, draft prepared by the Séminaire Paul Sally, 1995, Available at www.math.ubc.ca/ cass/research/publications.html

[GK75] I. M. Gel'fand and D. A. Kajdan, Representations of the group $\mathrm{GL}(n, K)$ where $K$ is a local field, Lie groups and their representations (Proc. Summer School, Bolyai János Math. Soc., Budapest, 1971), Halsted, New York, 1975, pp. 95-118. MR0404534

[HH98] A. G. Helminck and G. F. Helminck, A class of parabolic $k$-subgroups associated with symmetric k-varieties, Trans. Amer. Math. Soc. 350 (1998), no. 11, 4669-4691, DOI 10.1090/S0002-9947-98-02029-7. MR.1443876

[HR90] Michael J. Heumos and Stephen Rallis, Symplectic-Whittaker models for $\mathrm{Gl}_{n}$, Pacific J. Math. 146 (1990), no. 2, 247-279. MR1078382

[HW93] A. G. Helminck and S. P. Wang, On rationality properties of involutions of reductive groups, Adv. Math. 99 (1993), no. 1, 26-96, DOI 10.1006/aima.1993.1019. MR.1215304

[JR96] Hervé Jacquet and Stephen Rallis, Uniqueness of linear periods, Compositio Math. 102 (1996), no. 1, 65-123. MR1394521

[KS17] F. Knop and B. Schalke, The dual group of a spherical variety, Trans. Moscow Math. Soc. 78 (2017), 187-216, DOI 10.1090/mosc/270. MR3738085

[KT08] Shin-ichi Kato and Keiji Takano, Subrepresentation theorem for p-adic symmetric spaces, Int. Math. Res. Not. IMRN 11 (2008), Art. ID rnn028, 40, DOI 10.1093/imrn/rnn028. MR2428854

[KT10] Shin-ichi Kato and Keiji Takano, Square integrability of representations on $p$ adic symmetric spaces, J. Funct. Anal. 258 (2010), no. 5, 1427-1451, DOI 10.1016/j.jfa.2009.10.026. MR2566307

[Lag08] Nathalie Lagier, Terme constant de fonctions sur un espace symétrique réductif p-adique (French, with English and French summaries), J. Funct. Anal. 254 (2008), no. 4, 10881145, DOI 10.1016/j.jfa.2007.07.012. MR2381204

[Off17] Omer Offen, On parabolic induction associated with a p-adic symmetric space, J. Number Theory 170 (2017), 211-227, DOI 10.1016/j.jnt.2016.06.014. MR.3541705

[OS07] Omer Offen and Eitan Sayag, On unitary representations of $\mathrm{GL}_{2 n}$ distinguished by the symplectic group, J. Number Theory 125 (2007), no. 2, 344-355, DOI 10.1016/j.jnt.2006.10.018. MR.2332593 
[OS08a] Omer Offen and Eitan Sayag, Global mixed periods and local Klyachko models for the general linear group, Int. Math. Res. Not. IMRN 1 (2008), Art. ID rnm 136, 25, DOI 10.1093/imrn/rnm136. MR2417789

[OS08b] Omer Offen and Eitan Sayag, Uniqueness and disjointness of Klyachko models, J. Funct. Anal. 254 (2008), no. 11, 2846-2865, DOI 10.1016/j.jfa.2008.01.004. MR2414223

[Rob83] Alain Robert, Introduction to the representation theory of compact and locally compact groups, London Mathematical Society Lecture Note Series, vol. 80, Cambridge University Press, Cambridge-New York, 1983. MR690955

[Rod73] François Rodier, Whittaker models for admissible representations of reductive p-adic split groups, Harmonic analysis on homogeneous spaces (Proc. Sympos. Pure Math., Vol. XXVI, Williams Coll., Williamstown, Mass., 1972), Amer. Math. Soc., Providence, R.I., 1973, pp. 425-430. MR0354942

[Smi18a] Jerrod Manford Smith, Local unitary periods and relative discrete series, Pacific J. Math. 297 (2018), no. 1, 225-256, DOI 10.2140/pjm.2018.297.225. MR.3864235

[Smi18b] Jerrod Manford Smith, Relative discrete series representations for two quotients of $p$ adic $\mathbf{G L}_{n}$, Canad. J. Math. 70 (2018), no. 6, 1339-1372, DOI 10.4153/CJM-2017-047-7. MR 3850546

[Smi20] Jerrod Manford Smith, The support of closed orbit relative matrix coefficients, manuscripta math. (2020).

[SV17] Yiannis Sakellaridis and Akshay Venkatesh, Periods and harmonic analysis on spherical varieties (English, with English and French summaries), Astérisque 396 (2017), viii+360. MR 3764130

[Tad85] Marko Tadić, Unitary dual of p-adic GL(n). Proof of Bernstein conjectures, Bull. Amer. Math. Soc. (N.S.) 13 (1985), no. 1, 39-42, DOI 10.1090/S0273-0979-1985-15355-8. MR788387

[Tad86] Marko Tadić, Classification of unitary representations in irreducible representations of general linear group (non-Archimedean case), Ann. Sci. École Norm. Sup. (4) 19 (1986), no. 3, 335-382. MR870688

[Xu17] Bin Xu, On Moglin's parametrization of Arthur packets for p-adic quasisplit $S p(N)$ and $S O(N)$, Canad. J. Math. 69 (2017), no. 4, 890-960, DOI 10.4153/CJM-2016-029-3. MR.3679701

[Zel80] A. V. Zelevinsky, Induced representations of reductive p-adic groups. II. On irreducible representations of $\mathrm{GL}(n)$, Ann. Sci. École Norm. Sup. (4) 13 (1980), no. 2, 165-210. MR.584084

Department of Mathematics and Statistics, University of Calgary, Calgary, AlBERTA, CANADA, T2N 1N4

Email address: jerrod.smith@ucalgary.ca 